\title{
Heat Capacity and Thermodynamic Properties of Poly(chlorotrifluoroethylene) from 2.5 to $620 \mathrm{~K}$
}

\section{Shu-Sing Chang and James J. Weeks $^{1}$ \\ National Institute of Standards and Technology, \\ Gaithersburg, MD 20899}

\author{
Heat capacities and thermodynamic \\ properties of a number of poly(chlorotri- \\ fluoroethylene) samples subjected to \\ various thermal treatments, to achieve \\ crystallinities ranging from approxi- \\ mately 10 to $90 \%$, have been studied \\ from 2.5 to $370 \mathrm{~K}$ by automated adia- \\ batic calorimetry and from 250 to $620 \mathrm{~K}$ \\ by differential scanning calorimetry. \\ Small heat capacity discontinuities in the \\ temperature range from 320 to $350 \mathrm{~K}$ \\ were observed in all samples with crys- \\ tallinities greater than $40 \%$. Sponta- \\ neous adiabatic temperature drifts \\ associated with these anomalies were \\ positive (exothermic) for quenched sam- \\ ples and negative (endothermic) for an- \\ nealed samplcs. Therefore these \\ anomalies were believed to be associ- \\ ated with a relaxation phenomenon simi- \\ lar to that of a glass transition. For \\ highly quenched low crystallinity films, a
}

much larger heat capacity discontinuity of greater than $15 \%$ was observed, amidst a crystallization exotherm. In addition to the above phenomena, annealing of the sample at any temperature between 240 to $400 \mathrm{~K}$ would produce a shift in the population distribution of crystallites from reorganization or melting and recrystallization. As a result, the apparent heat capacity became somewhat lowered at the annealing temperature and somewhat raised at about $20 \mathrm{~K}$ above the annealing temperature.

Key words: automated adiabatic calorimetry; crystallitc distribution; differential scanning calorimetry; glass transition; heat capacity; poly(chlorotrifluoroe thylene); residual entropy; thermodynamic properties.

Accepted: October 28, 1991

\section{Introduction}

Although poly(chlorotrifluoroethylene), PCTFE, is a fairly well-studied polymer in its physical and mechanical properties, its heat capacity and associated thermodynamic properties are only reported in a few publications [1-4] covering the temperature ranges: 2.5 to $20 \mathrm{~K}[2,4], 80$ to $340 \mathrm{~K}[3]$ and 273 to $515 \mathrm{~K}$ [1]. There are also thermal analytical studies concerned mainly with the melting and crystallization phenomena near the fusion region.

\footnotetext{
${ }^{1}$ Prcsent address: Route 1, Box 121, Mathias, WV 26812.
}

Glass transition phenomena were not observed by thermal means in the above mentioned publications. Dilatometric studies [5,6], however, have indicated the occurrence of a glass transition near $325 \mathrm{~K}$. Dielectric $[7,8]$ and mechanical relaxation [9] studies have also indicated a glass transitionlike relaxation together with crystalline relaxations.

Since the crystallinity of PCTFE can be changed over a wide range [6], the heat capacity behavior of three PCTFE samples of varied crystallinity and thermal treatments was studied in this work with a 
fully automated, high precision, adiabatic calorimeter [10], for the temperature range from 2.5 to 370 $\mathrm{K}$, and by a commercial differential scanning calorimeter from 250 to $620 \mathrm{~K}$. The precision of the adiabatic calorimeter, except at temperatures below $50 \mathrm{~K}$, was of the order of $0.02 \%$. The high sensitivity in the detection of spontaneous temperature changes, on the order of $10^{-5} \mathrm{~K}$, enabled the detection of the occurrence of broad relaxation phenomena, such as that occurring in polyethylene [11]. Techniques used in the adiabatic calorimetry enabled the separation of the underlying heat capacity contributed by lattice vibrations from that contributed by spontaneous thermal events occurring in the sample. The present work confirmed the existence of a glass-like relaxation in PCTFE, occurring in the temperature region from 320 to $350 \mathrm{~K}$. In the transition region, the spontaneous adiabatic temperature drifts were positive (exothermic) for quenched samples and negative (endothermic) for annealed samples.

Data from differential scanning calorimetry, covering a temperature range from 250 to $620 \mathrm{~K}$, were used to supplement the adiabatic calorimetry at temperatures above $370 \mathrm{~K}$. The fast thermal analysis operation enabled the study of a large number of samples with various thermal histories. In addition to the confirmation of the existence of the glass transition discontinuities in most samples studied, crystallization and melting were also studied. The glass transition occurs amidst a complicated crystalline reorganization phenomenon over a wide temperature range from 240 to $400 \mathrm{~K}$. By following rigorous calibration procedures, heat capacities and heats of fusion were obtained with reasonable precision using scanning calorimetry.

Combining the results from both adiabatic and scanning calorimetry, thermodynamic functions of the crystalline and the amorphous phases may be estimated. The amorphous phase contains a residual entropy at $0 \mathrm{~K}$.

\section{Experimental Detail \\ 2.1 Materials}

The adiabatic calorimetric samples of poly(chlorotrifluoroethylene), PCTFE, were composed of typical factory production KEL-F 81 brand plastics, KF-6060 pellet and KF-6061 powder, from Minnesota Mining and Manufacturing
Company. ${ }^{2}$ Both batches were of Grade 3 quality with the degree of polymerization of about 1600 , or a molecular weight of near 190,000. The molecular weight of the repeating monomeric unit, $-\mathrm{CClF}-\mathrm{CF}_{2}$-, is $116.47 \mathrm{~g} \mathrm{~mol}^{-1}$. Three samples were loaded into the adiabatic calorimeter: (1) KF6060 pellets as received, (2) high crystallinity sample prepared by slow crystallization from molten KF-6061 powder, and (3) quenched films made from KF-6061 powder. Table 1 lists loading conditions for the three adiabatic calorimetric samples, along with their initial densities $\rho_{i}$ and crystallinities $X_{\mathrm{i}}$. After the evacuation, a small amount of helium gas at a room temperature of about $296 \mathrm{~K}$ was sealed with the sample to aid the thermal conductivity. The amount of helium gas was equal to the weight of helium used when the empty sample container was measured.

In order to achieve a maximum degree of crystallinity, a slow melt-crystallized sample was prepared by heating KF-6061 powder in vacuum above the melting point to $525-535 \mathrm{~K}$, until all bubbles collapsed into a clear liquid. A motorized voltage source was used to slowly decrease the power to the heating mantle. The melt was cooled at a rate of 1-2 K per d, from 500 to about $400 \mathrm{~K}$.

To achieve the lowest degree of crystallinity, thin films of PCTFE of about $0.15 \mathrm{~mm}$ thickness were prepared by pressing the melt of KF-6061 powder between two aluminum plates at about $575-590 \mathrm{~K}$. The plates and the molten film were then quenched in icy water. In most of the films, the center portion of the film showed effects of air pockets and some anisotropic characteristics as the samples were viewed between crossed polarizers. Although the ranges of the densities of the clear

Table 1. Adiabatic calorimeter loading of PCTFE samples

\begin{tabular}{lcccc}
\hline \hline Designation & $\begin{array}{c}\text { Mass (vacuo) } \\
\mathrm{g}\end{array}$ & $\begin{array}{c}\rho_{\mathrm{i}} \\
\mathrm{g} \mathrm{cm}^{-3}\end{array}$ & $X_{\mathrm{i}}$ & $\begin{array}{r}\mathrm{He} \\
\mathrm{kPa}\end{array}$ \\
\hline X (Slow crystallized) & 124.617 & 2.170 & 0.87 & 4.2 \\
P (Pellet, as received) & 145.147 & 2.133 & 0.54 & 5.3 \\
F (Film) & 63.396 & 2.095 & 0.13 & 3.3 \\
\hline
\end{tabular}

\footnotetext{
${ }^{2}$ Certain commercial equipment, instruments, or materials are identified in this paper to specify adequately the experimental procedure. Such identification does not imply recommendation or endorsement by the National Institute of Standards and Technology, nor does it imply that the materials or equipment identified are necessarily the best available for the purpose.
} 
region and the anisotropic region were about the same at $2.095 \pm 0.005 \mathrm{~g} \mathrm{~cm}^{-3}$, in order for the calorimetric sample to retain as high an amorphous character as possible, the optically anisotropic regions were cut off and not used.

Initial crystallinities of the three samples were estimated from their densities as $0.13,0.54$, and 0.87 for the film, pellet, and melt-crystallized samples, respectively. The densities [6] of 2.076 and $2.186 \mathrm{~g} \mathrm{~cm}^{-3}$ for the amorphous and the crystalline phases, respectively, were used for the crystallinity estimation. For DSC measurements, besides the samples described above, the KF-6061 powder as received, quenched films made from $\mathrm{KF}-6060$ pellets, a manufactured PCTFE tubing of $0.11 \mathrm{~mm}$ thickness, and a low molecular weight PCTFE KEL-F 200 Wax were also used.

If the quenched films had not been exposed to $325 \mathrm{~K}$ or higher temperatures, the $\mathrm{x}$-ray $(\mathrm{CuK \alpha})$ diffraction patterns of these highly amorphous samples all showed an intense halo centered around a $2 \theta$ value of $15.5^{\circ}$ or around a spacing of $5.7 \AA$ plus two fainter broad halos at around $32^{\circ}$ and $39^{\circ}$ or around 2.8 and $2.3 \AA$, similar to that found in commercial PCTFE films [12,13]. A faint halo at $8^{\circ}$ or about $11 \AA$ was always observed. For crystalline PCTFE, regardless of thermal treatments and degrees of crystallinity, the most intense lines are located at $5.5,5.4,5.2$, and $4.9 \AA$, which are followed by two groups of fainter rings starting at 3.2 and $2.8 \AA$, as observed previously [14]. However, in all crystalline samples, there appeared also the halos at 11 and $2.3 \AA$. In addition, there was also a diffused ring at $14.8^{\circ}$ or $6.0 \AA$.

The $x$-ray pattern of the low molecular weight KEL-F 200 wax, above its melting point at $373 \mathrm{~K}$, appeared just like the amorphous film. At $409 \mathrm{~K}$, the major halo shifted to $15^{\circ}$ or the spacing increased to $5.9 \AA$. At room temperature, crystallized wax showed rings similar to that of the crystalline high molecular weight PCTFE, except the rings are more diffuse and somewhat difficult to resolve.

\subsection{Adiabatic Calorimetry}

A fully automated vacuum adiabatic calorimeter operating in the temperature range from 2 to $370 \mathrm{~K}$ [10] was used in this investigation. The calorimetric system included an automated analog adiabatic shield control system as improved from an earlier description [15], and a digital system for data acquisition and experimental control [10]. The analog control system was capable of maintaining the shield temperature and following the temperature changes of the sample container to within $1 \mathrm{mK}$. The principal measuring instruments in the digital system included a high resolution, self-balancing potentiometer [16] as modified from a commercially available manual potentiometer of a Diesselhorst ring [17] design, highly stable constant current sources, and a commercially available nanovolt amplifier with a linearity of $0.01 \%$. This system was capable of measuring $0.1 \mathrm{~V}$ at full-scale with a resolution of less than $10 \mathrm{nV}$. Therefore this system provided a temperature resolution of near $10^{-5} \mathrm{~K}$ at temperatures above $50 \mathrm{~K}$, from a nominal $25 \Omega$ platinum resistance thermometer operating at a current of 1-2 mA.

Above $14 \mathrm{~K}$, the temperature scale used was IPTS-1968 [18]. At lower temperatures, the platinum resistance thermometer was calibrated against a germanium thermometer which was in turn calibrated against the NBS provisional temperature scale 2-20 (1965) [19]. The temperature scale below $14 \mathrm{~K}$ may either be interpolated directly from the calibration data or by following a three point calibration procedure [20]. At temperatures below $273 \mathrm{~K}$, the scale IPTS-1968 differs from ITS-90 [21] by a maximum of $14 \mathrm{mK}$ in the temperature region of 130 to $160 \mathrm{~K}$. Between 273 and 373 $\mathrm{K}$, the size of the temperature unit changes by $0.025 \%$. The greatest change of $0.1 \%$ in the size of the temperature unit occurs at temperature regions of between $15-20 \mathrm{~K}$ and $50-60 \mathrm{~K}$. The changes in the size of the temperature unit directly affects the heat capacity value calculation. Since the changes were $0.1 \%$ or less, no corrections were made to change the temperature scale from IPTS-1968 to IPS-90.

The heat capacity, $C_{p}$, of the sample container assembly, including the sample, the container, the thermometer and the heater, was determined by the equilibrium temperature rise produced by an electrical energy input. The decay time constant toward the establishment of a temperature equilibrium after a disturbance was in the order of 100 $\mathrm{s}$ for this assembly [22]. Approximately $15 \mathrm{~min}$ were required after each energy input to reestablish a temperature equilibrium, or to reach a temperature drift on the order of or less than $0.1 \mathrm{mK}$ $\min ^{-1}$. The small drift arises from heat exchanges between the calorimeter and its surroundings due to a residual deviation in the control of a strict adiabatic condition. The time constant is much shorter at low temperatures due to the increase in the thermal conductivity of most materials. Much longer decay time constants with temperature drifts greater than that expected from the non-adiabatic 
conditions of the shield control system indicated the presence of a spontaneous thermal effect originating in the sample. Even under these adverse conditions, the instantaneous or short term heat capacities, contributed mainly from lattice vibrations, could still be estimated by extrapolating the drifts to obtain the temperature rise at the middle of an energy input. The energy of the spontaneous thermal event over a temperature range is obtainable by summing all energy inputs under adiabatic conditions and then subtracting from it the enthalpy increments as integrated from the lattice vibration heat capacity curve.

\subsection{Differential Scanning Calorimetry}

Some of the early heats of fusion and melting points were observed with a Perkin-Elmer DSC-1B differential scanning calorimeter. Most of the thermal analyses were performed with a Perkin-Elmer DSC-2 differential scanning calorimeter. The accuracy of the DSC measurements depended strongly on calibrations and the correctness of the assigned baseline for each scan. Meltings of indium and benzoic acid were used to calibrate the scales for the temperature and the power. Although scans of different rates were sometimes used, most of the runs were performed at a heating rate of $10 \mathrm{~K}$ $\min ^{-1}$. Due to the thermal conductivity of the system, the temperature of the sample capsule lags behind the temperature of the thermometer/sample holder assembly as a function of heating rates by about $0.06 \mathrm{~K}\left(\mathrm{~K} \mathrm{~min}^{-1}\right)^{-1}$. For better precision in heat capacity determinations, calibration scans of sapphire discs were frequently performed and the results compared against standard values $[23,24]$. For even better precision in heat capacity determinations, short scans with scanning temperature ranges of 5 to $10 \mathrm{~K}$ were used. Under these conditions, the uncertainty in the baseline is minimized. The average heat capacity for the 5 to $10 \mathrm{~K}$ interval may also be calculated by integrating the scan curve for the enthalpy and dividing the latter by the temperature interval, as in the intermittent heating method used in the adiabatic calorimetry. Heat capacities obtained by the short range method were found in agreement with adiabatic values to better than $0.5 \%$.

Although DSC scans were performed at much lower temperatures, only the data above $250 \mathrm{~K}$, where $C_{p}$ of PCTFE began to vary significantly with crystallinity, were used to supplement the adiabatic data.
One peculiar result was observed when heat capacities were calculated from continuous scans at temperatures above $500 \mathrm{~K}$. The heat capacity values from continuous scans became progressively higher than that from short scans by about $20 \%$ at $600 \mathrm{~K}$. Similar results were observed when the heat capacities of copper and teflon were measured. The reason for the occurrence of this discrepancy is unknown. It may be of instrumental nature or due to contamination or chemical reactions. Therefore long scans at high temperatures were used only for the observation of the melting points and the heat of fusion. The short scans were used for the heat capacity of the liquid at high temperatures.

\section{Results and Discussion \\ 3.1 Adiabatic Calorimetry}

The conditions and thermal histories before each series of adiabatic calorimetric measurements on the three samples are listed chronologically in Table 2. The results of the heat capacity measurements are listed in Table 3 and shown in different regions in Figs. 1 and 2. For clarity, not all series of measurements are shown in Fig. 1, as the results are close to each other below $250 \mathrm{~K}$. As mentioned previously, the heat capacity reported here from adiabatic calorimetry represents mainly the vibrational contribution, free from the influence of long term spontaneous drifts due to crystallization and glassy relaxation.

3.1.1 Heat Capacity At low temperatures, a Debye $T^{3}$ region was not observed in this investigation. The values of $C_{p} / T^{3}$ were still increasing rapidly for all samples even at $2.5 \mathrm{~K}$ as the temperature was lowered, see Fig. 3. This is characteristic of the presence of a large amount of disorder and soft mode of vibration in all PCTFE samples, regardless of their crystallinities as estimated by the density. The rise of $C_{p} / T^{3}$ to a peak, before a possible Debye region occurs at a lower temperature, has been observed generally in the temperature range of $2-5 \mathrm{~K}$ for almost all glassy materials. This peak occurs at $4 \mathrm{~K}$ for cis-1,4-polyisoprene [25], 3 $\mathrm{K}$ for amorphous selenium [26,27], 3-7 $\mathrm{K}$ for a number of thermoset resins as summarized in Ref. [28], and $3 \mathrm{~K}$ for polystyrene [29-31]. Similar to this investigation, the $C_{p} / T^{3}$ values have not yet reached a peak even at $2 \mathrm{~K}$ for glassy $o$-terphenyl [32] and poly(vinylchloride) [33]. Weaker peaks also exist in highly crystalline polyethylene [34], 
Table 2. Conditions and thermal histories of PCTFE

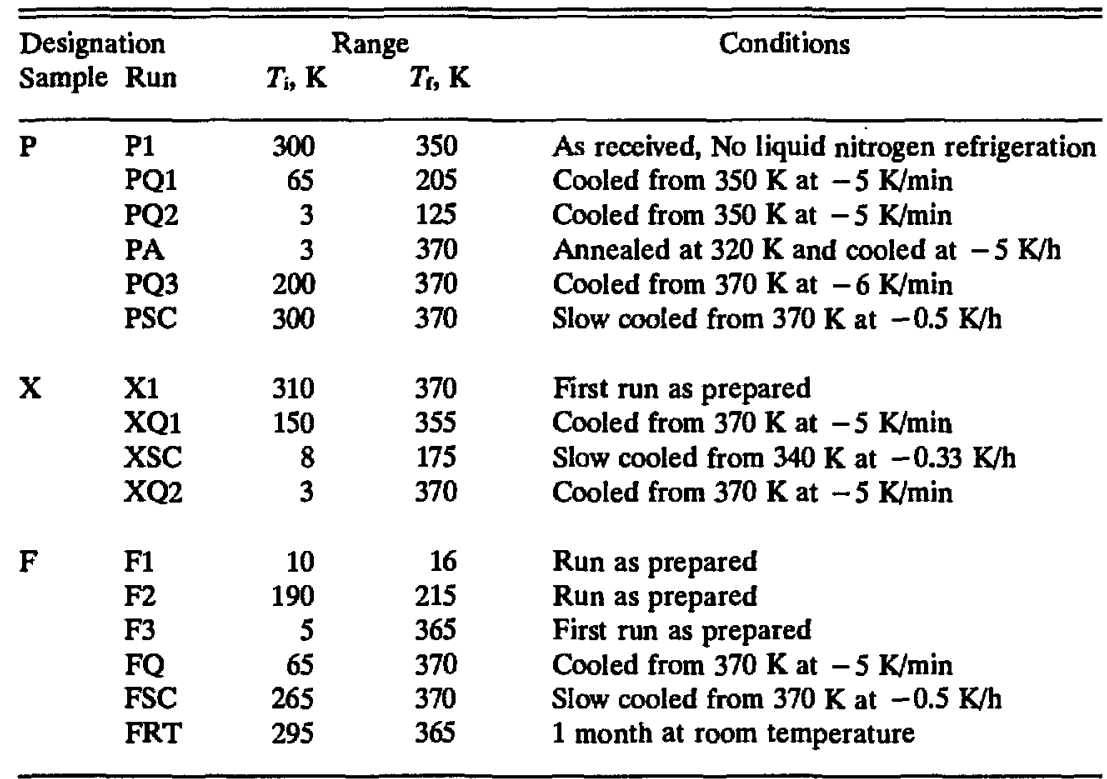

Table 3. Heat capacities of PCTFE ( $1 \mathrm{~mol}=116.47 \mathrm{~g})$

\begin{tabular}{|c|c|c|c|c|c|}
\hline $\begin{array}{l}T \\
\mathbf{K}\end{array}$ & $\begin{array}{c}C_{p} \\
\mathbf{J ~ K}^{-1} \mathrm{~mol}^{-1}\end{array}$ & $\begin{array}{l}T \\
\mathbf{K}\end{array}$ & $\begin{array}{c}C_{p} \\
\mathrm{~J} \mathrm{~K}^{-1} \mathrm{~mol}^{-1}\end{array}$ & $\begin{array}{l}T \\
\mathbf{K}\end{array}$ & $\underset{\mathrm{J} \mathrm{K}^{-1} \mathrm{~mol}^{-1}}{C_{p}}$ \\
\hline \multicolumn{6}{|c|}{ Pellet } \\
\hline \multicolumn{6}{|c|}{ P1 } \\
\hline 305.01 & 101.33 & 320.07 & 105.88 & 335.24 & 110.91 \\
\hline 309.98 & 102.81 & 325.08 & 107.39 & 340.28 & 112.80 \\
\hline 315.01 & 104.23 & 330.14 & 109.01 & 345.35 & 114.39 \\
\hline \multicolumn{6}{|c|}{ PQ1 } \\
\hline 65.40 & 26.66 & 112.28 & 45.49 & 161.94 & 62.24 \\
\hline 69.25 & 28.24 & 115.77 & 46.77 & 165.63 & 63.38 \\
\hline 71.68 & 29.21 & 119.28 & 48.06 & 169.27 & 64.48 \\
\hline 75.05 & 30.63 & 122.82 & 49.34 & 172.87 & 65.54 \\
\hline 78.44 & 32.03 & 126.29 & 50.56 & 176.52 & 66.63 \\
\hline 81.86 & 33.43 & 129.81 & 51.79 & 180.23 & 67.71 \\
\hline 85.17 & 34.80 & 133.36 & 53.02 & 183.90 & 68.78 \\
\hline 88.53 & 36.17 & 136.85 & 54.21 & 187.52 & 69.81 \\
\hline 91.88 & 37.53 & 140.39 & 55.39 & 191.21 & 70.85 \\
\hline 95.24 & 38.88 & 143.98 & 56.67 & 194.95 & 71.91 \\
\hline 98.61 & 40.21 & 147.51 & 57.70 & 198.65 & 72.94 \\
\hline 102.00 & 41.54 & 151.09 & 58.85 & 202.32 & 73.95 \\
\hline 105.41 & 42.87 & 154.72 & 60.00 & & \\
\hline 108.83 & 44.19 & 158.31 & 61.12 & & \\
\hline
\end{tabular}


Table 3. Heat capacities of PCTFE $(1 \mathrm{~mol}=116.47 \mathrm{~g})-$ Continued

\begin{tabular}{|c|c|c|c|c|c|}
\hline $\begin{array}{l}T \\
\mathrm{~K}\end{array}$ & $\underset{\mathrm{J} \mathrm{K}^{-1} \mathrm{~mol}^{-1}}{C_{p}}$ & $\begin{array}{l}T \\
\mathrm{~K}\end{array}$ & $\underset{\mathrm{J} \mathrm{K}^{-1} \mathrm{~mol}^{-1}}{C_{p}}$ & $\begin{array}{l}T \\
\mathrm{~K}\end{array}$ & $\underset{\mathrm{J} \mathrm{K}^{-1} \mathrm{~mol}^{-1}}{C_{p}}$ \\
\hline \multicolumn{6}{|c|}{ Pellet } \\
\hline \multicolumn{6}{|c|}{$\mathrm{PQ} 2$} \\
\hline 2.56 & 0.13 & 13.67 & 4.55 & 67.72 & 27.64 \\
\hline 2.60 & 0.15 & 14.98 & 5.21 & 72.11 & 29.44 \\
\hline 2.78 & 0.15 & 16.46 & 5.96 & 76.43 & 31.25 \\
\hline 3.15 & 0.22 & 18.18 & 6.83 & 80.80 & 33.15 \\
\hline 3.66 & 0.33 & 20.14 & 7.79 & 85.23 & 34.88 \\
\hline 4.24 & 0.46 & 22.46 & 8.91 & 89.64 & 36.70 \\
\hline 4.86 & 0.63 & 24.95 & 10.07 & 94.04 & 38.46 \\
\hline 5.52 & 0.83 & 27.63 & 11.27 & 98.45 & 40.23 \\
\hline 6.23 & 1.10 & 30.87 & 12.67 & 102.88 & 41.95 \\
\hline 6.99 & 1.40 & 34.60 & 14.23 & 107.32 & 43.70 \\
\hline 7.71 & 1.69 & 38.81 & 15.94 & 111.80 & 45.36 \\
\hline 8.43 & 2.02 & 43.86 & 17.96 & 116.32 & 47.08 \\
\hline 9.34 & 2.43 & 49.18 & 20.10 & 120.89 & 48.68 \\
\hline 10.42 & 2.94 & 54.07 & 22.08 & 125.51 & 50.48 \\
\hline 11.48 & 3.45 & 58.68 & 23.93 & & \\
\hline 12.50 & 3.97 & 63.21 & 25.80 & & \\
\hline \multicolumn{6}{|c|}{ PA } \\
\hline 2.77 & 0.18 & 83.24 & 34.08 & 230.24 & 81.30 \\
\hline 2.97 & 0.18 & 87.56 & 35.84 & 235.19 & 82.56 \\
\hline 3.47 & 0.28 & 91.95 & 37.63 & 240.07 & 83.87 \\
\hline 4.17 & 0.44 & 96.44 & 39.44 & 244.91 & 85.08 \\
\hline 4.97 & 0.67 & 100.94 & 41.19 & 249.80 & 86.28 \\
\hline 5.84 & 0.96 & 105.46 & 42.94 & 254.74 & 87.66 \\
\hline 6.82 & 1.33 & 110.00 & 44.71 & 259.63 & 88.87 \\
\hline 7.86 & 1.76 & 114.57 & 46.42 & 264.58 & 90.17 \\
\hline 8.90 & 2.23 & 119.10 & 48.07 & 255.46 & 87.54 \\
\hline 10.08 & 2.78 & 123.59 & 49.67 & 261.90 & 89.20 \\
\hline 11.31 & 3.37 & 128.14 & 51.28 & 266.53 & 90.57 \\
\hline 12.48 & 3.96 & 132.75 & 52.85 & 271.41 & 91.97 \\
\hline 13.77 & 4.60 & 137.42 & 54.48 & 276.34 & 93.37 \\
\hline 15.18 & 5.30 & 142.07 & 56.00 & 281.31 & 94.77 \\
\hline 16.69 & 6.08 & 146.71 & 57.52 & 286.34 & 96.01 \\
\hline 18.34 & 6.93 & 151.42 & 59.03 & 291.31 & 97.49 \\
\hline 21.02 & 8.22 & 156.14 & 60.46 & 296.24 & 98.88 \\
\hline 23.94 & 9.61 & 163.18 & 62.66 & 301.22 & 100.28 \\
\hline 26.66 & 10.85 & 168.93 & 64.32 & 306.24 & 101.59 \\
\hline 29.79 & 12.21 & 172.40 & 65.38 & 311.21 & 103.02 \\
\hline 33.07 & 13.60 & 177.21 & 66.79 & 316.24 & 104.26 \\
\hline 36.76 & 15.11 & 181.95 & 68.22 & 321.31 & 105.76 \\
\hline 41.08 & 16.86 & 186.73 & 69.56 & 326.33 & 107.12 \\
\hline 46.36 & 18.99 & 191.55 & 70.92 & 331.39 & 109.19 \\
\hline 51.76 & 21.18 & 196.32 & 72.23 & 336.47 & 111.28 \\
\hline 56.61 & 23.12 & 201.12 & 73.60 & 341.50 & 113.18 \\
\hline 61.22 & 24.98 & 205.98 & 74.90 & 346.56 & 114.76 \\
\hline 65.65 & 26.79 & 210.77 & 76.20 & 351.68 & 116.09 \\
\hline 70.08 & 28.64 & 215.62 & 77.48 & 356.75 & 117.35 \\
\hline 74.53 & 30.45 & 220.51 & 78.70 & 361.88 & 118.58 \\
\hline 78.91 & 32.31 & 225.35 & 80.03 & 367.07 & 119.75 \\
\hline
\end{tabular}


Table 3. Heat capacities of PCTFE (1 mol =116.47 g) - Continued

\begin{tabular}{|c|c|c|c|c|c|}
\hline $\begin{array}{l}T \\
\mathrm{~K}\end{array}$ & $\begin{array}{c}C_{p} \\
\mathrm{~J} \mathrm{~K}^{-1} \mathrm{~mol}^{-1}\end{array}$ & $\begin{array}{l}T \\
\mathrm{~K}\end{array}$ & $\underset{\mathrm{J} \mathrm{K}^{-1} \mathrm{~mol}^{-1}}{C_{p}}$ & $\begin{array}{l}T \\
\mathbf{K}\end{array}$ & $\begin{array}{c}C_{p} \\
\mathrm{~J} \mathrm{~K}^{-1} \mathrm{~mol}^{-1}\end{array}$ \\
\hline \multicolumn{6}{|c|}{ Pellet } \\
\hline \multicolumn{6}{|c|}{ PQ3 } \\
\hline 204.46 & 74.50 & 267.39 & 90.91 & 322.93 & 106.77 \\
\hline 208.76 & 75.64 & 272.36 & 92.29 & 325.90 & 107.75 \\
\hline 213.62 & 76.98 & 277.28 & 93.59 & 328.85 & 108.57 \\
\hline 218.54 & 78.24 & 282.25 & 94.95 & 331.78 & 109.61 \\
\hline 223.39 & 79.58 & 287.27 & 96.42 & 334.69 & 110.75 \\
\hline 228.20 & 80.83 & 292.24 & 97.83 & 337.59 & 111.74 \\
\hline 233.05 & 82.08 & 297.25 & 99.25 & 340.46 & 112.94 \\
\hline 237.96 & 83.35 & 302.31 & 100.72 & 343.38 & 114.00 \\
\hline 242.82 & 84.66 & 307.32 & 102.19 & 346.36 & 114.62 \\
\hline 247.73 & 85.80 & 311.26 & 103.31 & 350.50 & 115.66 \\
\hline 252.70 & 87.10 & 314.15 & 104.19 & 355.70 & 116.70 \\
\hline 257.61 & 88.34 & 317.02 & 104.99 & 360.94 & 118.23 \\
\hline 262.47 & 89.63 & 319.94 & 106.01 & 366.33 & 119.50 \\
\hline \multicolumn{6}{|c|}{ PSC } \\
\hline 302.13 & 100.43 & 328.07 & 107.62 & 351.37 & 115.91 \\
\hline 306.04 & 101.42 & 331.96 & 108.92 & 335.30 & 116.83 \\
\hline 309.92 & 102.54 & 335.80 & 110.66 & 359.29 & 117.85 \\
\hline 316.73 & 104.28 & 339.68 & 112.56 & 363.26 & 118.78 \\
\hline 320.46 & 105.50 & 343.60 & 113.89 & 367.20 & 119.72 \\
\hline 324.16 & 106.47 & 347.50 & 114.97 & & \\
\hline \multicolumn{6}{|c|}{ Slow melt-crystallized } \\
\hline \multicolumn{6}{|c|}{$\mathbf{X} 1$} \\
\hline 313.68 & 100.48 & 334.31 & 105.03 & 354.66 & 109.02 \\
\hline 319.20 & 101.78 & 339.45 & 105.98 & 359.76 & 109.98 \\
\hline 324.23 & 103.02 & 344.55 & 107.03 & 364.93 & 111.03 \\
\hline 329.24 & 104.00 & 349.62 & 107.95 & 370.06 & 112.10 \\
\hline \multicolumn{6}{|c|}{$\mathrm{XQ1}$} \\
\hline 154.40 & 60.16 & 219.90 & 79.19 & 288.89 & 95.25 \\
\hline 157.77 & 61.24 & 224.72 & 80.49 & 293.97 & 96.24 \\
\hline 162.51 & 62.70 & 229.62 & 81.73 & 299.01 & 97.33 \\
\hline 167.18 & 64.18 & 234.57 & 82.92 & 304.00 & 98.39 \\
\hline 17189 & 65.56 & 239.46 & 84.12 & 308.97 & 99.45 \\
\hline 176.65 & 67.03 & 244.31 & 85.38 & 313.99 & 100.54 \\
\hline 181.34 & 68.38 & 249.22 & 86.47 & 319.08 & 101.67 \\
\hline 186.08 & 69.82 & 254.19 & 87.61 & 324.23 & 102.85 \\
\hline 190.86 & 71.18 & 259.11 & 88.73 & 329.35 & 103.97 \\
\hline 195.59 & 72.56 & 264.00 & 89.81 & 334.42 & 104.99 \\
\hline 200.36 & 73.87 & 268.95 & 90.94 & 339.57 & 105.97 \\
\hline 205.21 & 75.23 & 273.97 & 91.99 & 344.67 & 107.03 \\
\hline 210.09 & 76.68 & 278.95 & 93.11 & 349.74 & 107.94 \\
\hline 215.02 & 77.98 & 283.89 & 94.14 & 354.78 & 108.95 \\
\hline
\end{tabular}


Table 3. Heat capacities of PCTFE $(1 \mathrm{~mol}=116.47 \mathrm{~g})-$ Continued

\begin{tabular}{|c|c|c|c|c|c|}
\hline $\begin{array}{l}T \\
\mathrm{~K}\end{array}$ & $\begin{array}{c}C_{p} \\
\mathrm{~J} \mathrm{~K}^{-1} \mathrm{~mol}^{-1}\end{array}$ & $\begin{array}{l}T \\
\mathrm{~K}\end{array}$ & $\begin{array}{c}C_{p} \\
\mathrm{~J} \mathrm{~K}^{-1} \mathrm{~mol}^{-1}\end{array}$ & $\begin{array}{l}T \\
\mathrm{~K}\end{array}$ & $\begin{array}{c}C_{p} \\
\mathrm{~J} \mathrm{~K}^{-1} \mathrm{~mol}^{-1}\end{array}$ \\
\hline \multicolumn{6}{|c|}{ Slow melt-crystallized } \\
\hline \multicolumn{6}{|c|}{$\mathrm{XSC}$} \\
\hline 8.09 & 1.83 & 37.63 & 15.71 & 117.00 & 47.45 \\
\hline 8.96 & 2.22 & 41.53 & 17.26 & 121.49 & 49.10 \\
\hline 10.28 & 2.86 & 60.86 & 25.04 & 125.99 & 50.73 \\
\hline 11.49 & 3.46 & 64.76 & 26.59 & 130.50 & 52.30 \\
\hline 12.70 & 4.07 & 68.71 & 28.20 & 135.04 & 53.88 \\
\hline 14.00 & 4.74 & 73.02 & 30.00 & 139.60 & 55.40 \\
\hline 15.41 & 5.49 & 77.35 & 31.78 & 144.19 & 56.93 \\
\hline 16.95 & 6.29 & 81.73 & 33.58 & 148.81 & 58.41 \\
\hline 18.63 & 7.16 & 86.17 & 35.40 & 153.47 & 59.90 \\
\hline 20.48 & 8.09 & 90.54 & 37.18 & 158.16 & 61.38 \\
\hline 22.53 & 9.10 & 94.58 & 38.91 & 162.90 & 62.86 \\
\hline 24.81 & 10.20 & 99.27 & 40.66 & 167.56 & 64.28 \\
\hline 27.39 & 11.37 & 103.66 & 42.39 & 172.26 & 65.73 \\
\hline 30.33 & 12.66 & 108.03 & 44.07 & & \\
\hline 33.72 & 14.11 & 112.52 & 45.78 & & \\
\hline \multicolumn{6}{|c|}{ XQ2 } \\
\hline 3.02 & 0.19 & 92.59 & 38.08 & 247.92 & 86.21 \\
\hline 3.69 & 0.31 & 97.01 & 39.83 & 252.90 & 87.41 \\
\hline 4.55 & 0.52 & 101.45 & 41.60 & 257.83 & 88.51 \\
\hline 5.57 & 0.83 & 105.92 & 43.30 & 262.72 & 89.49 \\
\hline 6.70 & 1.24 & 110.43 & 45.06 & 267.68 & 90.61 \\
\hline 7.92 & 1.75 & 114.98 & 46.77 & 272.71 & 91.65 \\
\hline 9.19 & 2.33 & 119.58 & 48.45 & 277.70 & 92.76 \\
\hline 10.46 & 2.94 & 124.15 & 50.11 & 282.64 & 93.80 \\
\hline 11.68 & 3.54 & 128.59 & 51.58 & 287.56 & 94.81 \\
\hline 12.88 & 4.17 & 133.18 & 53.19 & 292.44 & 95.84 \\
\hline 14.19 & 4.83 & 137.90 & 54.81 & 297.38 & 96.87 \\
\hline 15.62 & 5.59 & 142.53 & 56.36 & 302.39 & 97.91 \\
\hline 17.18 & 6.40 & 147.18 & 57.89 & 306.36 & 98.74 \\
\hline 18.88 & 7.29 & 151.86 & 59.40 & 309.39 & 99.48 \\
\hline 20.69 & 8.19 & 156.58 & 60.95 & 312.32 & 100.10 \\
\hline 22.62 & 9.14 & 166.01 & 63.90 & 315.16 & 100.67 \\
\hline 24.84 & 10.20 & 170.73 & 65.38 & 318.06 & 101.41 \\
\hline 27.40 & 11.37 & 175.49 & 66.77 & 320.95 & 102.29 \\
\hline 30.23 & 12.61 & 180.18 & 68.22 & 323.82 & 102.73 \\
\hline 33.43 & 13.98 & 184.93 & 69.58 & 326.68 & 103.38 \\
\hline 37.24 & 15.55 & 189.72 & 70.98 & 329.54 & 103.95 \\
\hline 41.71 & 17.32 & 194.45 & 72.30 & 332.45 & 104.55 \\
\hline 47.01 & 19.44 & 199.23 & 73.65 & 335.44 & 105.12 \\
\hline 52.32 & 21.56 & 204.07 & 74.96 & 338.41 & 105.79 \\
\hline 57.09 & 23.51 & 208.85 & 76.27 & 341.37 & 106.44 \\
\hline 61.76 & 25.41 & 213.68 & 77.59 & 347.49 & 107.42 \\
\hline 66.26 & 27.24 & 218.57 & 78.96 & 350.99 & 108.23 \\
\hline 70.64 & 29.04 & 223.41 & 80.17 & 354.89 & 108.97 \\
\hline 74.95 & 30.82 & 228.31 & 81.40 & 358.77 & 109.75 \\
\hline 79.32 & 32.68 & 233.26 & 82.66 & 362.63 & 110.52 \\
\hline 83.76 & 34.47 & 238.16 & 83.84 & 366.06 & 111.22 \\
\hline 88.18 & 36.25 & 243.01 & 85.16 & 369.93 & 112.08 \\
\hline
\end{tabular}


Table 3. Heat capacities of PCTFE ( $1 \mathrm{~mol}=116.47 \mathrm{~g})$-Continued

\begin{tabular}{|c|c|c|c|c|c|}
\hline $\begin{array}{l}T \\
\mathbf{K}\end{array}$ & $\underset{\mathrm{J} \mathrm{K}^{-1} \mathrm{~mol}^{-1}}{c}$ & $\begin{array}{l}T \\
\mathrm{~K}\end{array}$ & $\underset{\mathrm{J} \mathrm{K}^{-1} \mathrm{~mol}^{-1}}{C_{n}}$ & $\begin{array}{l}T \\
\mathrm{~K}\end{array}$ & $\underset{\mathrm{J} \mathrm{K}^{-1} \mathrm{~mol}^{-1}}{C_{p}}$ \\
\hline \multicolumn{6}{|c|}{ Quenched film } \\
\hline \multicolumn{6}{|c|}{ F1 } \\
\hline 9.95 & 2.72 & 12.25 & 3.83 & 14.86 & 5.12 \\
\hline 11.00 & 3.22 & 13.50 & 4.45 & 16.34 & 5.84 \\
\hline \multicolumn{6}{|c|}{ F2 } \\
\hline 190.74 & 70.46 & 200.22 & 73.07 & 209.90 & 75.73 \\
\hline 195.51 & 71.80 & 205.02 & 74.40 & 214.74 & 77.02 \\
\hline \multicolumn{6}{|c|}{ F3 } \\
\hline 4.79 & 0.62 & 109.73 & 44.52 & 275.89 & 93.98 \\
\hline 5.72 & 0.93 & 114.20 & 46.18 & 280.78 & 95.65 \\
\hline 6.86 & 1.36 & 118.75 & 47.86 & 285.75 & 97.17 \\
\hline 8.09 & 1.87 & 123.29 & 49.50 & 290.68 & 98.75 \\
\hline 9.36 & 2.44 & 127.82 & 51.09 & 295.57 & 100.46 \\
\hline 10.63 & 3.03 & 132.46 & 52.71 & 299.45 & 101.93 \\
\hline 11.84 & 3.61 & 137.11 & 54.26 & 302.34 & 103.34 \\
\hline 13.06 & 4.21 & 141.76 & 55.73 & 304.94 & 104.92 \\
\hline 14.37 & 4.84 & 146.43 & 57.29 & 307.25 & 106.62 \\
\hline 15.80 & 5.56 & 151.12 & 58.72 & 309.65 & 107.40 \\
\hline 17.36 & 6.32 & 155.84 & 60.26 & 312.05 & 108.33 \\
\hline 19.06 & 7.15 & 165.25 & 63.10 & 314.45 & 109.72 \\
\hline 20.92 & 8.03 & 169.91 & 64.51 & 316.92 & 110.87 \\
\hline 22.99 & 9.01 & 174.64 & 65.86 & 319.37 & 112.15 \\
\hline 27.86 & 11.19 & 179.32 & 67.26 & 321.83 & 113.52 \\
\hline 30.57 & 12.34 & 184.07 & 68.62 & 324.27 & 114.73 \\
\hline 33.64 & 13.64 & 188.91 & 69.98 & 326.71 & 116.28 \\
\hline 37.30 & 15.15 & 193.69 & 71.29 & 329.14 & 117.82 \\
\hline 41.61 & 16.87 & 198.41 & 72.61 & 331.58 & 119.69 \\
\hline 46.77 & 18.98 & 203.10 & 73.86 & 334.02 & 122.34 \\
\hline 51.99 & 21.10 & 207.86 & 75.16 & 336.51 & 122.43 \\
\hline 56.65 & 23.01 & 212.71 & 76.51 & 338.94 & 122.52 \\
\hline 61.09 & 24.81 & 217.51 & 77.78 & 341.29 & 122.99 \\
\hline 65.52 & 26.66 & 222.27 & 79.09 & 343.69 & 123.22 \\
\hline 69.98 & 28.49 & 227.11 & 80.43 & 346.16 & 123.88 \\
\hline 74.38 & 30.34 & 232.04 & 81.73 & 348.69 & 123.51 \\
\hline 78.73 & 32.18 & 236.93 & 82.97 & 351.19 & 124.13 \\
\hline 83.08 & 34.00 & 241.77 & 84.21 & 353.60 & 124.84 \\
\hline 87.44 & 35.74 & 246.56 & 85.90 & 355.99 & 124.99 \\
\hline 91.82 & 37.53 & 251.44 & 86.94 & 358.37 & 125.34 \\
\hline 96.24 & 39.29 & 256.41 & 88.31 & 360.73 & 125.86 \\
\hline 100.71 & 41.06 & 261.34 & 89.69 & 363.60 & 126.71 \\
\hline 105.24 & 42.89 & 266.23 & 91.15 & & \\
\hline
\end{tabular}


Table 3. Heat capacities of PCTFE $(1 \mathrm{~mol}=116.47 \mathrm{~g})$-Continued

\begin{tabular}{|c|c|c|c|c|c|}
\hline $\begin{array}{l}T \\
\mathbf{K}\end{array}$ & $\begin{array}{c}C_{p} \\
\mathrm{~J} \mathrm{~K}^{-1} \mathrm{~mol}^{-1}\end{array}$ & $\begin{array}{l}T \\
\mathrm{~K}\end{array}$ & $\begin{array}{c}C_{p} \\
\mathrm{~J} \mathrm{~K}^{-1} \mathrm{~mol}^{-1}\end{array}$ & $\begin{array}{l}T \\
\mathbf{K}\end{array}$ & $\underset{\mathrm{J} \mathrm{K}^{-1}}{C_{p}} \mathrm{~mol}^{-1}$ \\
\hline \multicolumn{6}{|c|}{ Quenched film } \\
\hline \multicolumn{6}{|c|}{ FQ } \\
\hline 66.73 & 27.16 & 160.29 & 61.64 & 266.24 & 91.06 \\
\hline 70.56 & 28.72 & 165.02 & 63.09 & 274.10 & 92.39 \\
\hline 74.84 & 30.51 & 169.83 & 64.53 & 276.03 & 93.89 \\
\hline 78.99 & 35.25 & 174.57 & 65.93 & 281.06 & 95.46 \\
\hline 83.25 & 33.99 & 183.88 & 68.60 & 286.04 & 97.01 \\
\hline 87.62 & 35.77 & 188.59 & 69.99 & 290.98 & 98.61 \\
\hline 91.95 & 37.54 & 193.37 & 71.32 & 295.87 & 100.21 \\
\hline 96.26 & 39.27 & 198.11 & 72.61 & 300.85 & 101.87 \\
\hline 100.71 & 41.02 & 202.92 & 73.92 & 305.90 & 103.60 \\
\hline 105.16 & 42.75 & 207.82 & 75.23 & 310.91 & 105.22 \\
\hline 109.62 & 44.45 & 212.68 & 76.54 & 315.88 & 106.98 \\
\hline 114.09 & 46.13 & 217.48 & 77.86 & 320.93 & 108.37 \\
\hline 118.59 & 47.79 & 222.24 & 79.09 & 326.07 & 110.15 \\
\hline$₫ 23.13$ & 49.41 & 227.09 & 80.41 & 331.15 & 111.83 \\
\hline 127.68 & 51.01 & 232.03 & 81.60 & 336.20 & 113.57 \\
\hline 132.28 & 52.60 & 236.92 & 82.96 & 341.21 & 115.42 \\
\hline 136.91 & 54.18 & 241.76 & 84.39 & 346.29 & 117.49 \\
\hline 141.61 & 55.73 & 246.56 & 85.65 & 351.44 & 119.65 \\
\hline 146.22 & 57.23 & 251.44 & 86.92 & 356.53 & 121.85 \\
\hline 150.89 & 58.71 & 256.42 & 88.26 & 361.57 & 123.83 \\
\hline 155.63 & 60.18 & 261.35 & 89.59 & 366.69 & 125.52 \\
\hline \multicolumn{6}{|c|}{ FSC } \\
\hline 267.79 & 90.94 & 301.99 & 101.85 & 337.06 & 112.99 \\
\hline 272.40 & 92.70 & 307.03 & 103.54 & 342.05 & 114.98 \\
\hline 277.34 & 94.01 & 312.03 & 105.09 & 347.12 & 117.55 \\
\hline 282.23 & 95.54 & 316.99 & 106.74 & 352.35 & 120.70 \\
\hline 287.20 & 97.03 & 322.04 & 108.22 & 357.66 & 122.66 \\
\hline 292.13 & 98.71 & 327.04 & 109.75 & 362.79 & 124.53 \\
\hline 297.02 & 100.22 & 332.01 & 111.30 & 367.89 & 125.98 \\
\hline \multicolumn{6}{|c|}{ FRT } \\
\hline 296.81 & 100.06 & 319.78 & 107.85 & 343.70 & 116.07 \\
\hline 298.74 & 100.81 & 321.79 & 108.46 & 346.53 & 117.38 \\
\hline 300.63 & 101.43 & 323.73 & 109.10 & 349.45 & 119.00 \\
\hline 302.51 & 102.15 & 325.66 & 109.69 & 351.51 & 119.86 \\
\hline 304.46 & 102.96 & 327.59 & 110.23 & 335.46 & 120.86 \\
\hline 306.39 & 103.51 & 329.46 & 110.72 & 355.45 & 122.06 \\
\hline 308.38 & 104.22 & 331.43 & 111.42 & 357.43 & 122.91 \\
\hline 310.30 & 104.66 & 333.45 & 112.07 & 359.46 & 123.61 \\
\hline 312.17 & 105.41 & 335.41 & 112.70 & 361.48 & 124.05 \\
\hline 314.08 & 106.10 & 337.41 & 113.47 & 363.49 & 124.72 \\
\hline 315.99 & 106.64 & 339.67 & 113.50 & & \\
\hline 317.89 & 107.17 & 341.77 & 114.87 & & \\
\hline
\end{tabular}




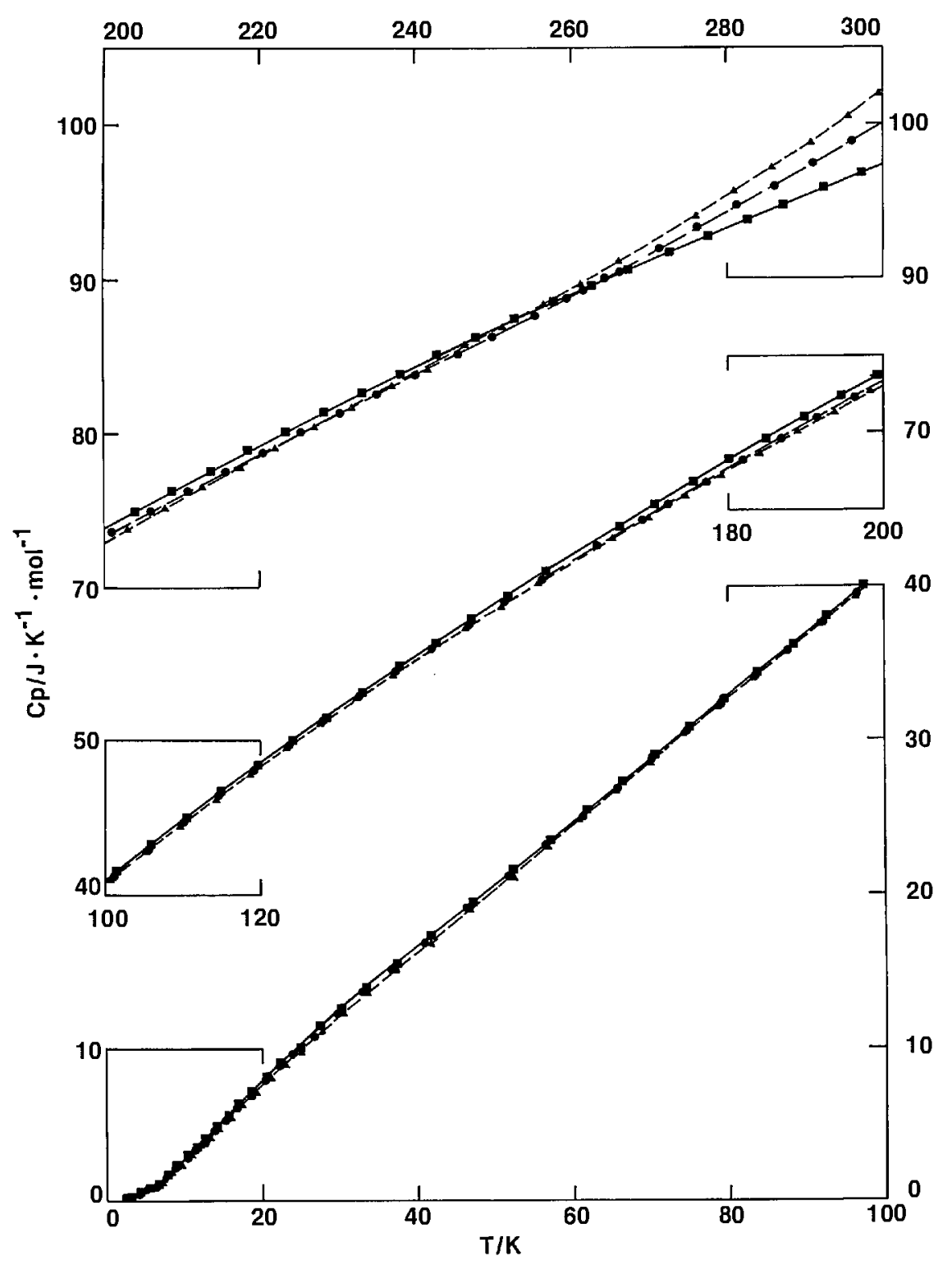

Fig. 1. Heat capacity of PCTFE.

Film: A. Pellet: 0 . Slow crystallized:

crystalline $o$ - and $p$-terphenyl $[32,35]$ and even in a hard crystalline material such as sapphire [23].

There are two publications $[2,4]$ reporting the cryogenic behavior of PCTFE. From thermal conductivity measurements of a sample of PCTFE with a density of $2.114 \mathrm{~g} \mathrm{~cm}^{-3}$ from 1 to $4.5 \mathrm{~K}$ [2], an average value for $C / T^{3}$ of $0.123 \mathrm{~mJ} \mathrm{~K}^{-4} \mathrm{~cm}^{-3}$ or about $6.8 \mathrm{~mJ} \mathrm{~K}^{-4} \mathrm{~mol}^{-1}$ was given. This value is approximately the average of the values observed in the present study, see Fig. 3.

The results of specific heat measurements for a sample of PCTFE without detailed description from 2.5 to $20 \mathrm{~K}$ are reported in several ways [4], which are also shown in Fig. 3 for comparison. The graphical data were estimated from a $\log (C)$ $\log (T)$ plot for $T<10 \mathrm{~K}$ and from a $C / T-T^{2}$ plot for $T>10 \mathrm{~K}$ from their publication. The data at $20 \mathrm{~K}$ was also obtained from the $\log -\log$ plot, as the $T^{2}$ plot only extended to $18 \mathrm{~K}$. Only in the region between 6 to $14 \mathrm{~K}$, do the graphical data agree reasonably well with our data. Below $5 \mathrm{~K}$, the graphical data indicate a lowering of $C / T^{3}$ from 4.6 $\mathrm{mJ} \mathrm{K}^{-4} \mathrm{~mol}^{-1}$ at $5 \mathrm{~K}$ to $3.7 \mathrm{~mJ} \mathrm{~K}^{-4} \mathrm{~mol}^{-1}$ at $2.5 \mathrm{~K}$. The value at $2.5 \mathrm{~K}$ was nearly one-half of that from this study and that from a thermal conductivity study [2]. The authors, however, assigned a much higher Debye constant of $5.6 \mathrm{~mJ} \mathrm{~K}^{-4} \mathrm{~mol}^{-1}$, which is much higher than their graphical data, but is still 


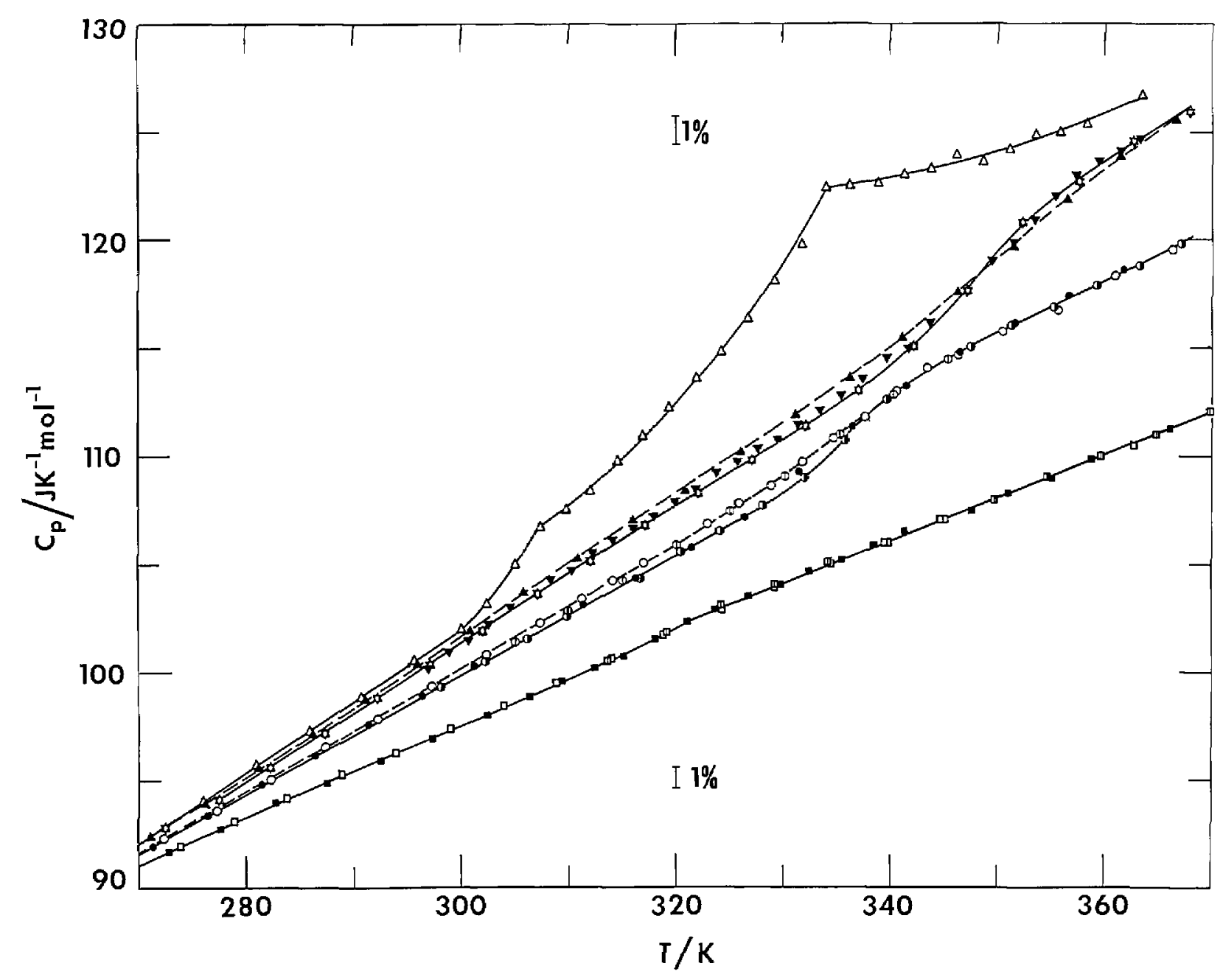

Fig. 2. Heat capacity of PCTFE and glass transition.

Film: $\triangle-F 3, \Delta-F Q, \nabla-F S C$, FRT.

Pellet: O-P1, (D-PQ3, O-PA, -PSC.

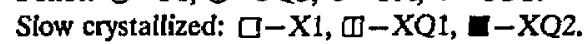

lower than that in the present study. The authors also assigned a constant for a $C / T^{2.5}$ dependency for the range 4.3 to $6.7 \mathrm{~K}$, which yielded values much closer to the values of the present study. The reasons for the different representations and the discrepancies among their graphical data and assigned constants are unknown.

It may also be noted that a mixture of PCTFE grease in silica gel was reported [36] to have heat capacities almost five times higher than the values reported by us and in Ref. [2] for PCTFE alone, in the temperature range from 1.8 to $4.5 \mathrm{~K}$.

A region of $C_{p}$ proportionality to $T$ exists between 30 and $100 \mathrm{~K}$, as seen in Figs. 1 and 4. The heat capacity function, $C_{p} / T$, curves in Fig. 4 also included a few DSC scans as discussed in later sections. Above $100 \mathrm{~K}, C_{p}$ of PCTFE still assumes a relatively linear dependency on temperature, as seen by slowly varying $C_{p} / T$ values. The linear dependency and proportionality may be a result of a relatively uniform distribution of vibrational frequencies from 100 to $1000 \mathrm{~cm}^{-1}$, as estimated by Guttman [37].

From 100 to $260 \mathrm{~K}$, heat capacities of all samples studied are surprisingly close to each other, within a spread of about $1 \%$, regardless of a large variation in sample densities or crystallinities. The spread is greatest, about $3 \%$, around $30 \mathrm{~K}$. However, over the wide temperature region from 10 to $250 \mathrm{~K}$, the heat capacity is higher for samples of higher crystallinity. Generally the $C_{p}$ of most glassy materials is higher than the $C_{p}$ of their crystalline counterpart. Although examples have been cited [38] to show that the $C_{p}$ of the crystalline state is 
Journal of Research of the National Institute of Standards and Technology

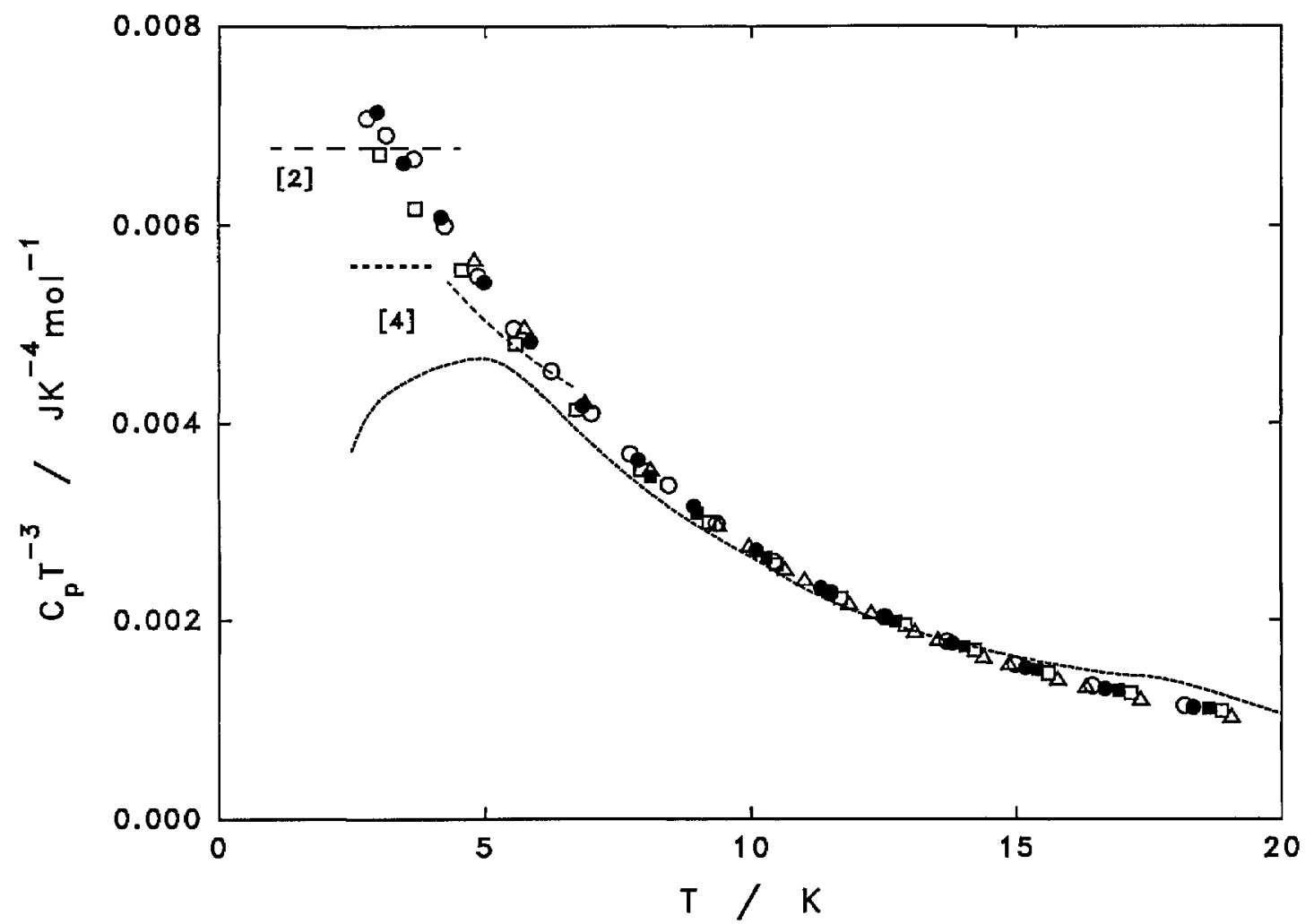

Fig. 3. Low temperature heat capacity of PCTFE.

Film: $\triangle-F 1, F 3$.

Pellet: O-PQ2, -PA.

Slow crystallized: $\square-\mathrm{XQ2}, \mathbf{\square}-\mathrm{XSC}$.

Long dashes - Ref. [2]. Short dashes -Ref. [4].

higher than that of the vitreous state in certain ranges of temperature, the occurrence of this phenomenon over such a wide temperature range is rather unusual.

Above $260 \mathrm{~K}$, the heat capacity depends strongly on the crystallinity or the complementary amorphous content. The lower the amorphous content, the lower the heat capacity, as normally expected of most semicrystalline materials.

Heat capacities of PCTFE above the cryogenic temperatures were only reported in two publications $[1,3] . C_{p}$ values between 80 and $340 \mathrm{~K}$ of two PCTFE samples with crystallinities of 0.46 and 0.65 , as determined by densities, were studied and smoothed data on the high density sample reported [3]. The reported values agree very well with that for our pellet sample series $\mathrm{P} 1$ and $\mathrm{PQ}$. Similar to our findings, the authors found that the heat capacities for the two samples were the same within their experimental error of $0.3 \%$ at temperatures below $325 \mathrm{~K}$. Their highest temperature measurement stops in the glass transition region, just as the transition is about to be completed. The authors failed to detect or report a glass transition, except to note an indication of a positive $\mathrm{d}^{2} C_{p} / \mathrm{d} T^{2}$. At 340 $\mathrm{K}$, the heat capacity of the low density sample was about $0.8 \%$ higher than that of the denser sample.

Two samples of PCTFE, with crystallinities of 0.35 and 0.82 as determined from the heat of fusion, were studied with a differential calorimeter in a continuous heating mode for the temperature region from 0 to $250^{\circ} \mathrm{C}$ [1]. No glass transition was reported for either of the two samples. The results from these two samples will be compared with DSC studies of the present work in later sections of this paper.

3.1.2 Glass Transition and Tacticity A glasslike transition was observed in all adiabatic calorimetric samples in the region from 320 to $350 \mathrm{~K}$, as shown in Fig. 2. The intensity, as viewed by the magnitude of the sudden heat capacity rise, $\Delta C_{p}$, is a function of the amorphous content. In the transition region, the spontaneous adiabatic temperature drifts were positive (exothermic) for quenched samples and negative (endothermic) for annealed samples. Although there appeared a small $C_{p}$ dis- 


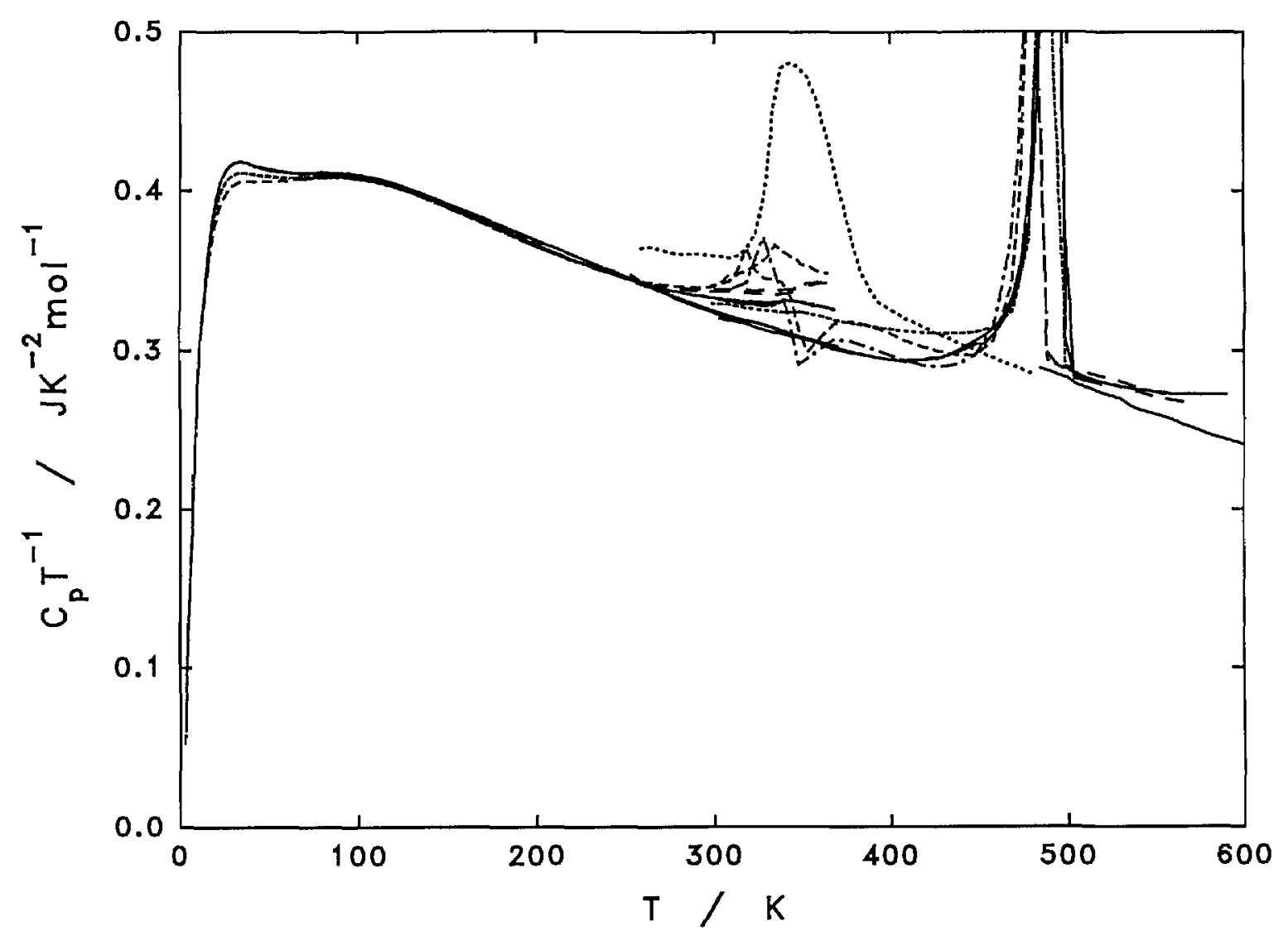

Fig. 4. Heat capacity function of PCTFE (adiabatic calorimetry and DSC). Solid lines - slow crystallized. Short dashes-pellet. Medium dashes-film (F1-F3). Long dashes-film (FAQ, FAS, FRT).

Dots - wax.

continuity at just below $320 \mathrm{~K}$ for the highly crystalline sample $X$, the drifts were all within $1 \mu \mathrm{K} \mathrm{s}^{-1}$ after 15-20 min following the termination of energy input. Figure 5 shows the spontaneous drifts for the pellet sample $P$, with the annealing peak occurring at a higher temperature than the quenched peak.

Figure 6 shows the drift behavior for the film sample F. For the first heating of highly amorphous films, there appeared an endothermic dip in the drift at about $307 \mathrm{~K}$. This may be a result of being left standing at the room temperature of $296 \mathrm{~K}$ for some time, after the films were quenched from the melt in ice water. The true drift peak for the glass transition probably was masked by the larger exothermic drifts due to crystallization occurring at temperatures above $310 \mathrm{~K}$. Subsequent to the crystallization and stabilization of the films for a long time at $370 \mathrm{~K}$, normal drifts for the glass transition phenomenon appeared in the temperature range of 340-350 K, see Fig. 6 . The heat capacity behavior of the film after the stabilization process lay in be- tween that of the pellet and the first heating of the films, see Fig. 2.

The variation of the glass transition of PCTFE as a function of crystallinity contradicts the commonly perceived idea that the glass transition temperature increases with crystallinity. Except for the highly amorphous quenched film, where the glass transition may be very wide and the true center of the glass transition was not observed, $T_{\mathrm{g}}$ appears to be lower for lower amorphous content. One plausible explanation for this phenomenon is that in the highly crystalline sample $X$, the remaining $10 \%$ (approximately) of amorphous content consists of mainly uncrystallizable low tactic or atactic portions of the polymer, and therefore the glass transition temperature appears near the low end of the glass transition range, as observed in the highly amorphous quenched film $\mathrm{F}$.

The variation of the glass transition temperature as a function of crystallinity is shown in Fig. 7, where results from dilatometric $[7,39]$ and dielectric [7] studies are also included. Earlier dilatomet- 


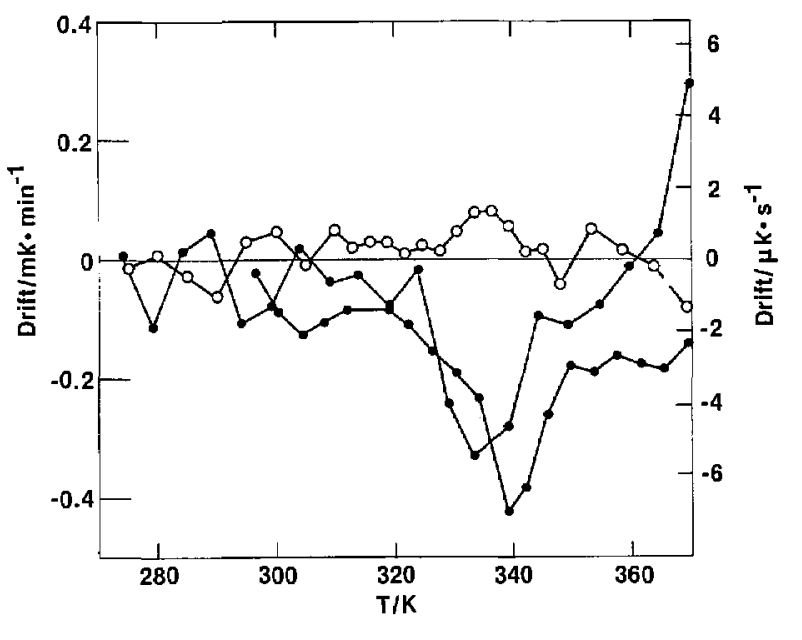

Fig. 5. Adiabatic temperature drift of pellet. O-quenched. . -annealed.

ric studies [6] of samples with densities of 2.116 and $2.165 \mathrm{~g} \mathrm{~cm}^{-3}$ suggested a glass transition temperature of $325 \mathrm{~K}$ which was relatively invariant with crystallinity.

\subsection{Differential Scanning Calorimetry}

About fifty DSC samples were taken from PCTFE samples of the same batches as the samples for adiabatic calorimetry, plus the original powder, films made from pellets, a manufactured tubing, and a low molecular weight wax. These DSC samples were subjected to various thermal treatments to show the dependency on the thermal history, such as annealing, quenching, and slow cooling. The influence of mechanical deformation was also studied. It is not feasible to show all the results of some 300 DSC runs in any detail except to make a summary here. A few of the typical runs, especially the first heatings of quenched samples, are shown as heat capacity functions in Fig. 4, as well as heat capacity curves in Fig. 11 in a later section. Except for the low molecular weight wax, the results on all other PCTFE samples, whether they were composed of pellet, powder or manufactured tubing, showed nearly identical behaviors as dictated by the thermal history after melting.

The low molecular weight Kel-F 200 Wax, showed a broad melting region from 305 to $400 \mathrm{~K}$, with a peak at $350 \mathrm{~K}$ and a heat of fusion of $15 \pm 1$ $\mathrm{J}^{-1}$. The units of per gram instead of per mole are used here for convenience. The liquid heat capacity appeared to connect reasonably well (within 1\%) to the adiabatic data for quenched film above its $T_{\mathrm{g}}$ and is slightly lower than the DSC liquid heat capacity above the $T_{\mathrm{m}}$ of the high molecular weight PCTFE. Upon cooling, crystallization starts around $375 \mathrm{~K}$ with a exothermic maximum at about $352-$ $355 \mathrm{~K}$. The wax showed some instability at temperatures above $510 \mathrm{~K}$.

The following summarizes the DSC observations on various forms of pellet, powder and film samples with different thermal histories. The observations included glass transition, crystallization, crystalline reorganization and fusion.

3.2.1 Glass Transition Temperature Except for the highly crystalline sample $\mathrm{X}$ where the small $\Delta C_{p}$ is difficult to observe by DSC, glass transition temperatures as depicted by a $C_{p}$ discontinuity were also observed by DSC in the temperature range of $330-360 \mathrm{~K}$, in similar magnitudes of $\Delta C_{p}$ as that observed by adiabatic calorimetry. The observation on the sample $P$ was shown in Fig. 4 as an example. The slight increase in the observed glass transition temperature is due to the kinetic nature of the glass transition and the dynamic nature of the DSC measurement.

In order to improve the thermal conductivity and thus the consistency of sample temperature with the indicated DSC temperature, film samples were scanned as they were immersed in silicone oil. The $T_{B}$ of films held overnight at $370 \mathrm{~K}$ was found to be in the range of 355 to $360 \mathrm{~K}$. Only if the cooling rate was below $0.1 \mathrm{~K} / \mathrm{min}$ or if the sample was annealed at $340 \mathrm{~K}$ for some time, did the transition range became narrower with a small relaxation peak at 360 to $375 \mathrm{~K}$. Samples cooled at higher rates show a broader transition range of about $\mathbf{3 0}$ $\mathrm{K}$.

For high amorphous content samples, apparent endothermic peaks occurred at about $320 \mathrm{~K}$ for the first heating of film sample $F$ and films quenched from molten pellets KF-6060. A manufactured thin tubing of $0.11 \mathrm{~mm}$ thickness, left at room temperature for about 18 years, was scanned to reveal an even larger endothermic peak at $329 \mathrm{~K}$, see Figs. 4 and 11 . The onset of these endothermic peaks were the result of the rapid rise of $C_{p}$ in the glass transition region, as seen by the adiabatic calorimetry, where spontaneous effects and crystallization effects were largely eliminated to reveal the underlying instantaneous lattice heat capacities.

However the nature of these peaks was rather complicated. The complexity could arise from a variety of origins, such as the glassy relaxation peak, the crystalline reorganization peak as described in a later section, or the termination of the $C_{p}$ rise by the onset of the crystallization exotherm. 


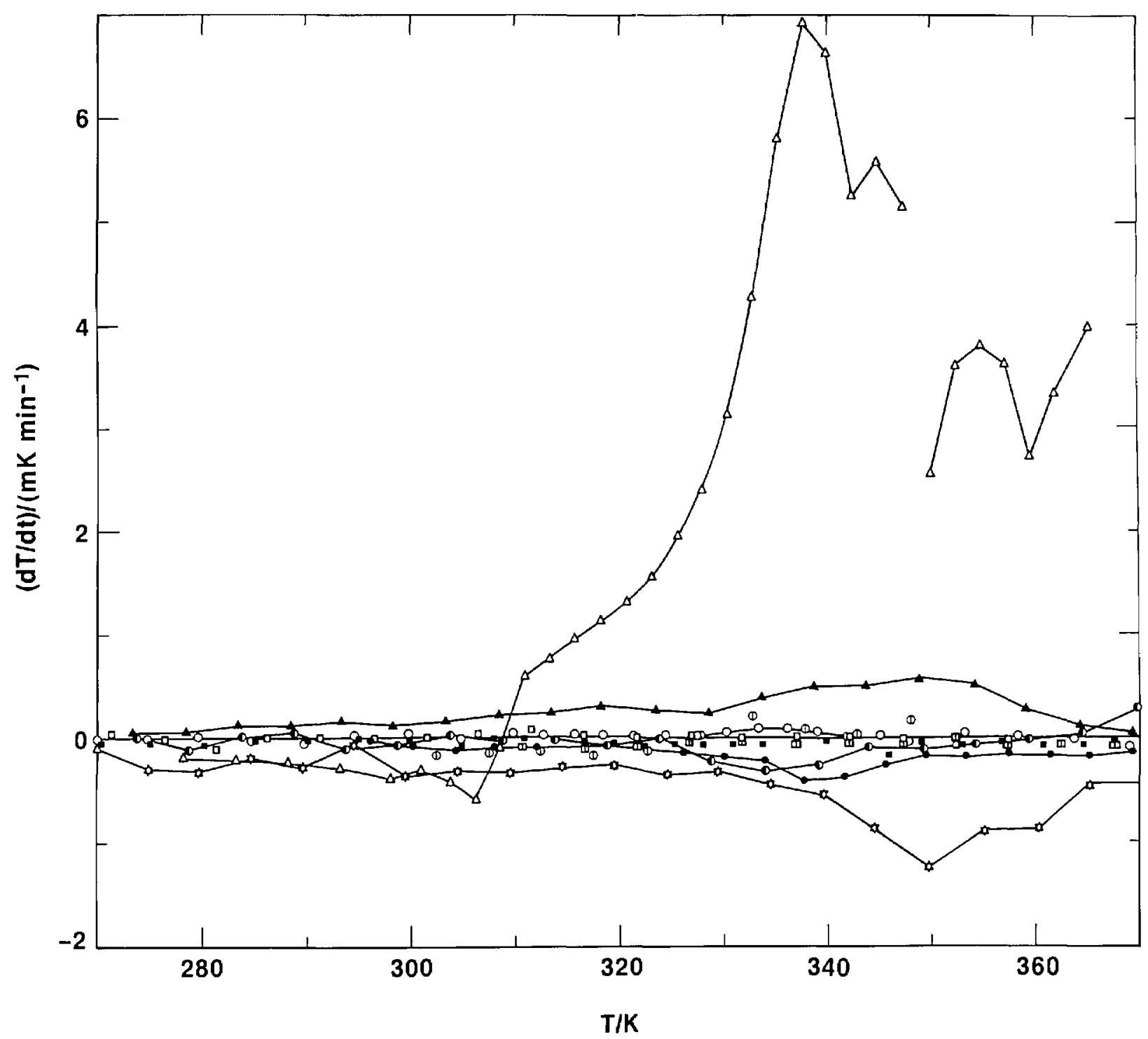

Fig. 6. Adiabatic temperature drift of film. Film: $\triangle-F 3, \Delta-F Q, \nabla-F S C$, $\Delta R T$.

Pellet: O-P1, $1-P Q 3,0-P A, 0-P S C$.

Slow crystallized: $\square-\mathrm{X} 1, \mathrm{\square}-\mathrm{XQ1}, \mathbf{\square}-\mathrm{XQ2}$.

3.2.2 Crystallization Crystallization of quenched films observed at a scan rate of $10 \mathrm{~K}$ $\min ^{-1}$ started near $335 \mathrm{~K}$ with an exothermic maximum at about $355 \mathrm{~K}$, Figs. 4 and 11, regardless of whether the film was quenched from the melt to room temperature water, icy water, or an acetonedry ice mixture at $195 \mathrm{~K}$. The crystallization exotherm for the films was on the order of $3 \mathrm{~J} \mathrm{~g}^{-1}$. Upon further heating, melting points for the first heating of quenched films were observed at 481$482.5 \mathrm{~K}$ with heats of fusion of $10.5-12.7 \mathrm{~J} \mathrm{~g}^{-1}$. The slower adiabatic calorimetry indicated the onset of crystallization for the quenched films near $310 \mathrm{~K}$ with a maximum rate near $340 \mathrm{~K}$ by the observations of adiabatic temperature drifts, Fig. 6.

A manufactured PCTFE tubing of $0.11 \mathrm{~mm}$ wall thickness, left at room temperature for 18 years, showed a crystallization onset at about $325 \mathrm{~K}$ and a maximum at about $350 \mathrm{~K}$ with a heat of crystallization of about $5 \mathrm{~J} \mathrm{~g} \mathrm{~g}^{-1}$ during the first heating. Further heating produced a melting point at $484 \mathrm{~K}$ with a heat of fusion of $16.3 \mathrm{~J} \mathrm{~g}^{-1}$. After cooling at $-10 \mathrm{~K} \mathrm{~min}^{-1}$ from the melt with a heat of crystallization of $19 \mathrm{~J} \mathrm{~g} \mathrm{~g}^{-1}$, the melting point is increased to $488 \mathrm{~K}$ with a heat of fusion of $19.3 \mathrm{~J} \mathrm{~g}^{-1}$. Although during the first heating of the tubing, it 


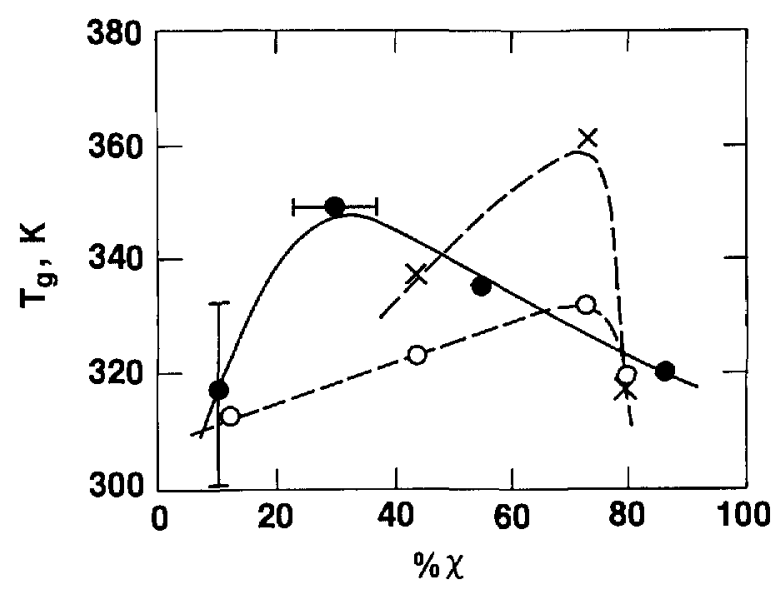

Fig. 7. Glass transition temperature as a function of crystallinity. -this work. O-dilatometric $[7,36]$. $x$-dielectric [7].

indicated a greater extent of crystallization than the films that we prepared, after the melting, the tubing behaved rather similarly to the samples made from either the pellets or from the powder.

The heat of crystallization of highly quenched films was much less than the subsequent heat of fusion. Therefore these highly amorphous films and the thin tubing were not completely amorphous but initially contained a certain crystallinity.

A film made from the powder, heated to $633 \mathrm{~K}$ and then quenched in water at room temperature, was studied for any stress-induced crystallization. It however behaved similar to films without any mechanical treatment. A strip of the film was drawn to break at about $50 \%$ elongation at room temperature. The $6 \mathrm{~mm}$ strip formed a neck of about 4 $\mathrm{mm}$ in width. A DSC scan of the neck area indicated a relatively large peak at $311 \mathrm{~K}$, followed by a crystallization exotherm with a maximum at $352 \mathrm{~K}$. The crystallization continued at temperatures above $410 \mathrm{~K}$. The sample was cooled at $440 \mathrm{~K}$. Upon reheating the sample, crystallization started again at $465 \mathrm{~K}$. This sample also showed reorganizational peaks at 316 and $354 \mathrm{~K}$, for annealing at room temperature and $335 \mathrm{~K}$, respectively. The melting point of this sample was at $487 \mathrm{~K}$. The untreated film from the same batch showed reorganizational peaks of 310 and $345 \mathrm{~K}$ for room temperature and 345 annealing, a crystallization exotherm maximum at $356 \mathrm{~K}$ and a melting point of $483 \mathrm{~K}$.

3.2.3 Reorganization of Crystallites The melting range for PCTFE is very wide, indicating a wide distribution of crystallite sizes and perfection. If a partially crystalline sample, obtained by a smooth continuous cooling procedure, was left standing at any temperature above $240 \mathrm{~K}$ for some time, the DSC thermogram would show a small dip at the annealing temperature and a small endothermic peak at about $20 \mathrm{~K}$ above the annealing temperature, Fig. 8. The dip in the DSC curve was not an exothermic phenomenon, but was caused by a slight depletion of the crystallite population that might be melting in the temperature region of concern. The small peak just above the annealing temperature was the result of the melting of the additional crystallite population from a previously existing smooth distribution. The small humps from annealing at temperatures above $400 \mathrm{~K}$ are more difficult to detect due to the superimposition onto the rapid rise of the thermogram of the main melting peak.

A more dramatic demonstration of this phenomenon was to create a sample with multiple small melting peaks. A film sample was first annealed at $400 \mathrm{~K}$ overnight and then cooled to lower temperatures at a rate of $10 \mathrm{~K} / \mathrm{min}$. The smooth cooling procedure was successively interrupted and the sample was annealed at 360,320 , and $280 \mathrm{~K}$ for $2.5 \mathrm{~h}$, and finally at $240 \mathrm{~K}$ overnight. Upon heating of this sample, small humps were observed at 268 , $298,341,377$, and $430 \mathrm{~K}$, e.g., at about 20 to $30 \mathrm{~K}$ above the annealing temperatures. Figure 8 shows the results from this demonstration, as well as from many observations with a single annealing temper-

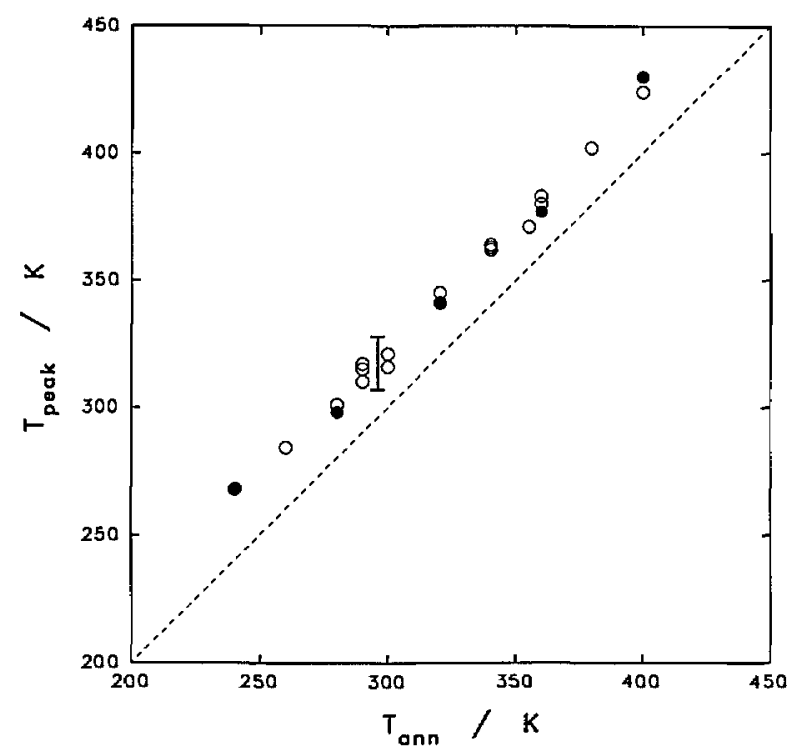

Fig. 8. Reorganization peak temperature as a function of annealing temperature.

- multiple peaks. O-individual peaks.

I-room temperature annealing. 
ature. Non-smooth distributions of crystallite population in partially crystalline polymers with a wide (over $100 \mathrm{~K}$ ) range of melting leading to multiple melting peaks have been observed in other polymers $[40,41]$.

Both the position and the intensity of this reorganization peak were also functions of annealing time, Fig. 9. The reorganizational peak disappears if the sample is re-scanned immediately after a smooth cooling process. For samples crystallized at $400 \mathrm{~K}$ and then annealed at a room temperature of $296 \mathrm{~K}$, the peak temperature varied from 307 to $320 \mathrm{~K}$ by annealing for a few minutes to several months. The peak height, as judged from the rescan, rose quickly with annealing time and then apparently leveled off after about $10 \mathrm{~h}$ at a level near $0.02 \mathrm{~J} \mathrm{~K}^{-1} \mathrm{~g}^{-1}$ or $2 \mathrm{~J} \mathrm{~K}^{-1} \mathrm{~mol}^{-1}$.

The increase of the peak temperature of the original film (including the manufactured tubing) appeared to have the same functionality as the increase of the peak temperature of the film after crystallization at $400 \mathrm{~K}$. The commercially produced PCTFE tubing of $0.11 \mathrm{~mm}$ thickness yielded a peak at $328 \mathrm{~K}$ after standing at room temperature for 18 years. However, the growth of the much larger peak by annealing the originally quenched film at room temperature was rather different from that of the crystallized film. The peak height varied from $0.05 \mathrm{~J} \mathrm{~K}^{-1} \mathrm{~g}^{-1}$ for annealing overnight at room temperature and to about $0.2 \mathrm{~J} \mathrm{~g}^{-1}$ after

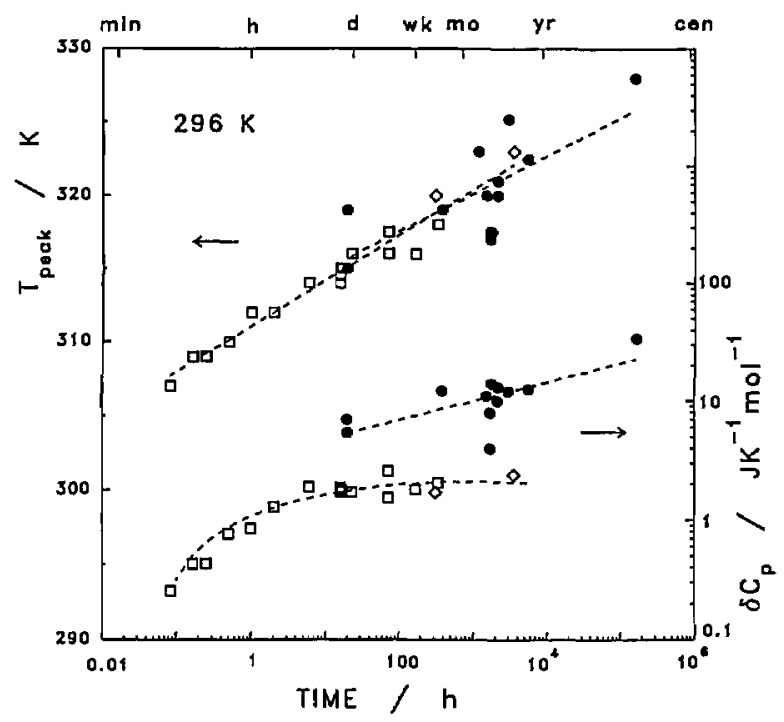

Fig. 9. Reorganization peak temperature and intensity as functions of annealing time.

$\square, \diamond-$ quenched films after crystallized at $400 \mathrm{~K}$.

- original films and tubing before heating to $400 \mathrm{~K}$. room temperature storage for $18 \mathrm{yr}$. It is possible that some of the peak height is contributed by the relaxation phenomena of the glass transition.

3.2.4 Melting Point and Heat of Fusion The melting temperature of PCTFE was not well defined and the major portion of the melting occurred over a wide temperature region of over 50 $K$. The usual practice for the determination of a sharp melting point in power-compensated type of DSC, such as the instrument used here, is to estimate the onset temperature. The temperature difference between the peak and the onset for a sharp first order transition is a function of the sample size. However, the peak temperature for the broad melting of PCTFE was found to be relatively insensitive to the heating rate and the amount of sample used in the sample capsule. Therefore the peak temperature is chosen as the representative melting point, $T_{\mathrm{m}}$, for the sample.

For low crystallinity samples consisting of quenched films or manufactured tubing, additional crystallization occurred above $335 \mathrm{~K}$ as the samples were scanned for the first time to their melting points. Melting points and heats of fusion for the first heating of quenched film were observed at $481-482.5 \mathrm{~K}$ with heats of fusion of $10.5-12.7 \mathrm{~J} \mathrm{~g}^{-1}$. The tubing gave a $T_{\mathrm{m}}$ of $484 \mathrm{~K}$ with $\Delta H_{\mathrm{m}}$ of $16.3 \mathrm{~J}$ $\mathrm{g}^{-1}$. As the energy release during the crystallization was about 3-5 $\mathrm{J} \mathrm{g}^{-1}$, the original film or tubing before the additional crystallization would have yielded a heat of fusion of 7-11 $\mathrm{J} \mathrm{g}^{-1}$.

Cooling the melt, regardless of the sample origin, at a rate of $5-40 \mathrm{~K} \mathrm{~min}^{-1}$ produces $T_{\mathrm{m}}$ of around $486-489 \mathrm{~K}$ and a heat of fusion around $15-$ $20 \mathrm{~J} \mathrm{~g}^{-1}$, similar to most of the pellet or powder PCTFE samples measured in as-received condition. Samples fast-cooled in DSC at an indicated rate of $320 \mathrm{~K} \mathrm{~min}^{-1}$ also yielded $T_{\mathrm{m}}$ around $487 \mathrm{~K}$, without the $315 \mathrm{~K}$ peak and the subsequent recrystallization at around $355 \mathrm{~K}$. Thus the true cooling rate in the DSC was far less than that indicated.

For slow-crystallized samples, the melting points observed were about $494 \pm 1 \mathrm{~K}$ with a heat of fusion on the order of $29-30 \mathrm{~J} \mathrm{~g}^{-1}$. A sample held at $470 \mathrm{~K}$ overnight also gave a $T_{\mathrm{m}}$ of $492 \mathrm{~K}$. The heat of fusion from DSC, as estimated by integrating the peak area above a baseline drawn from the solidus heat capacity at about $430 \mathrm{~K}$ to the liquidus heat capacity at about $500 \mathrm{~K}$, is a lower bound of the value. The results for the heat of fusion and melting point measurements for various PCTFE samples are shown in Fig. 10. 


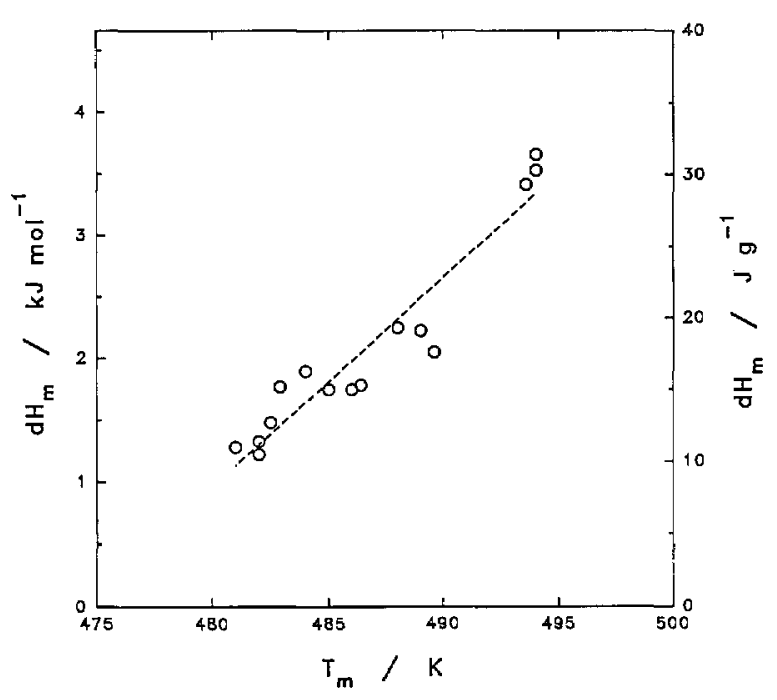

Fig. 10. Heat of fusion and melting point of PCTFE.
Based on the densities, the results of DSC measurements could only be extrapolated to about $35 \mathrm{~J}$ $\mathrm{g}^{-1}$ for the heat of fusion for the pure crystalline phase. A correction to the peak area, as based on the heat capacities of the crystalline and liquid phases as described in a later section and Fig. 11, would increase the heat of fusion value of the pure crystalline phase by $5 \mathrm{~J} \mathrm{~g}^{-1}$. The observed value for heat of fusion may be a lower bound for bulk crystals, due to the surface energy contributions from small crystallite sizes in these samples. We believe that a heat of fusion of about $40 \pm 5 \mathrm{~J} \mathrm{~g}^{-1}$ for the pure crystalline phase at an equilibrium melting point of $497 \mathrm{~K}$ [42] is perhaps a reasonable estimate.

Reference [1] reported heat of fusion values of $15.1 \pm 0.4 \mathrm{~J} \mathrm{~g} \mathrm{~g}^{-1}$ for air-quenched PCTFE and $35.1 \pm 0.8 \mathrm{~J} \mathrm{~g}^{-1}$ for a slow-cooled sample. The degrees of crystallinity for the two samples were esti-

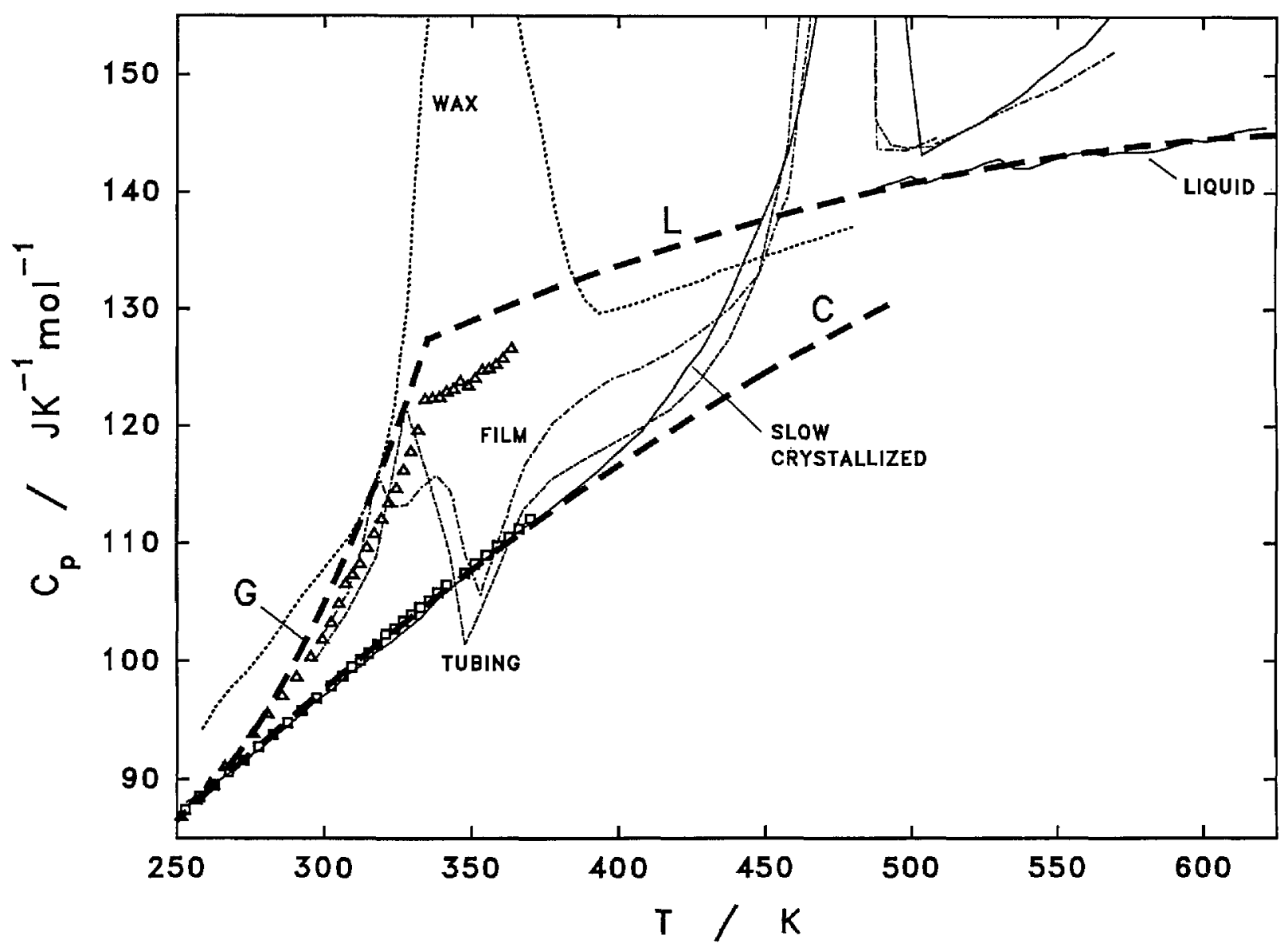

Fig. 11. Estimated heat capacity behavior of PCTFE crystal, glass, and liquid.

Heavy dashes - estimated heat capacity for PCTFE crystal (C), glass (G), and liquid (L).

Adiabatic measurements: $\triangle-F 3, \square-\mathrm{XQ2}$.

DSC measurements: wax, film (first heating), tubing (first heating), slow-crystallized and liquid (short scan) as labeled. 
mated as 0.35 and 0.82 , respectively, by using a value of $43.1 \pm 2.5 \mathrm{~J} \mathrm{~g}^{-1}$ as the heat of fusion of "pure" crystalline PCTFE from solubility studies [43]. Reference [1] also gave a value of $46.9 \pm 4.2 \mathrm{~J}$ $\mathrm{g}^{-1}$ as the heat of fusion for the pure crystals estimated by a method briefly described as volumetric [44]. A value of $75.7 \mathrm{~J} \mathrm{~g}^{-1}$ was reported for the heat of fusion by another solubility study [45], which we judged to be too high in comparison to all other studies.

Using the heat of fusion of the pure crystalline phase as the basis, the quenched film or the tubing would have a crystalline content of about $25 \%$ before any additional crystallization set in at temperatures above $350 \mathrm{~K}$. These residual crystallinities were small crystallites which were not detected by optical means and only gave a broad halo in x-ray powder patterns.

\subsection{Thermodynamic Properties}

3.3.1 Heat Capacity of PCTFE Crystal, Glass, and Liquid In the determination of the high temperature heat capacity of PCTFE by DSC, a peculiar behavior was found. At temperatures above $500 \mathrm{~K}$, heat capacities estimated from continuous scanning runs as calibrated with similar runs with sapphire discs became progressively higher than that obtainable by the short interval $(5-10 \mathrm{~K})$ scan or integration. This peculiar behavior was observed not only with the current PCTFE samples but also on heat capacity estimations of samples of teflon and copper, when compared with literature values. All results indicated deviations between the continuous scan and short interval scan of less than $0.5 \%$ at $430 \mathrm{~K}$ that increased to about $5 \%$ at $550 \mathrm{~K}$. The values measured by the short interval integration method on copper or teflon were however near $0.2 \%$ of the literature values throughout the entire temperature range. Therefore the short interval integration values are considered to be close to the true heat capacity values, and the long or continuous scans are to be used only for the estimation of heats of fusion and melting points. The reason for this discrepancy is unknown. It may be due to instrumental artifacts or due to the deterioration or contamination of the sample holder assembly, and/ or chemical reactions at high temperatures.

The heat capacity of crystalline PCTFE below $300 \mathrm{~K}$ is considered to be the same as that measured for the highly crystalline sample X, Table 4 . As the differences between the glassy and crystalline phases are rather small below $300 \mathrm{~K}$, no adjustment is applied to adjust for the near $10 \%$ amorphous content. Above $300 \mathrm{~K}, C_{p}$ of the crystalline phase is represented by a curve as shown in Fig. 11, which is a smooth extension of the heat capacity behavior below $300 \mathrm{~K}$. There is a slight reduction in the $C_{p}$ corresponding to the contribution from approximately $10 \%$ amorphous phase to the $\Delta C_{p}$ for the glass transition.

The heat capacity of amorphous PCTFE in the glassy state below $250 \mathrm{~K}$ is considered to be the same as the quenched film before heating to temperatures above the room temperature. As the differences between the glassy and crystalline phases are rather small below $300 \mathrm{~K}$, no adjustment is applied for any crystalline content in the quenched films. Above $250 \mathrm{~K}$ and extending to the supercooled liquid state, the heat capacity of the amorphous PCTFE is obtained by adding $25 \%$ of the $\Delta C_{p}$ to the measured heat capacity of the quenched

Table 4. Thermodynamic functions of crystalline PCTFE (units in $\mathrm{J}, \mathrm{K}$, and $\mathrm{mol}$. $1 \mathrm{~mol}=116.47 \mathrm{~g}$ )

\begin{tabular}{|c|c|c|c|c|}
\hline $\begin{array}{l}T \\
\mathrm{~K}\end{array}$ & $\begin{array}{c}C_{p} \\
\mathrm{~J} \mathrm{~K}^{-1} \mathrm{~mol}^{-1}\end{array}$ & $\begin{array}{l}H-H_{0, x} \\
\mathrm{~J} \mathrm{~mol}^{-1}\end{array}$ & $\underset{\mathrm{J} \mathrm{K}^{-1} \mathrm{~mol}^{-1}}{S}$ & $\begin{array}{c}-\left(G-H_{0, x}\right) \\
\mathrm{J} \mathrm{mol}^{-1}\end{array}$ \\
\hline 5 & 0.65 & 0.92 & 0.25 & 0.33 \\
\hline 10 & 2.71 & 8.96 & 1.28 & 3.88 \\
\hline 15 & 5.26 & 28.83 & 2.86 & 14.07 \\
\hline 20 & 7.84 & 61.64 & 4.73 & 32.97 \\
\hline 30 & 12.43 & 163.95 & 8.82 & 100.50 \\
\hline 40 & 16.46 & 308.19 & 12.94 & 209.26 \\
\hline 50 & 20.48 & 492.90 & 17.04 & 359.17 \\
\hline 60 & 24.51 & 717.79 & 21.13 & 550.03 \\
\hline 70 & 28.60 & 983.28 & 25.22 & 781.76 \\
\hline 80 & 32.72 & 1289.8 & 29.30 & 1054.3 \\
\hline 90 & 36.82 & 1637.6 & 33.39 & 1367.8 \\
\hline 100 & 40.84 & 20260 & 37.48 & 1722.2 \\
\hline 120 & 48.43 & 2919.9 & 45.61 & 2553.3 \\
\hline 140 & 55.51 & 3961.8 & 53.63 & 3546.0 \\
\hline 160 & 61.96 & 5137.2 & 61.47 & 4697.2 \\
\hline 180 & 68.05 & 6437.9 & 69.12 & 6003.3 \\
\hline 200 & 73.82 & 7857.2 & 76.59 & 7460.7 \\
\hline 220 & 79.24 & 9388.4 & 83.88 & 9065.7 \\
\hline 240 & 84.27 & 11024 & 91.00 & 10815 \\
\hline 260 & 88.91 & 12757 & 97.93 & 12704 \\
\hline 273.15 & 91.78 & 13945 & 102.72 & 14113 \\
\hline 280 & 93.31 & 14579 & 105.01 & 14825 \\
\hline 298.15 & 97.22 & 16310 & 111.00 & 16785 \\
\hline 300 & 97.64 & 16489 & 111.60 & 16991 \\
\hline 320 & 101.81 & 18485 & 118.04 & 19288 \\
\hline 340 & 105.79 & 20561 & 124.33 & 21712 \\
\hline 360 & 109.61 & 22716 & 130.49 & 24260 \\
\hline 380 & 113.24 & 24945 & 136.52 & 26931 \\
\hline 400 & 116.71 & 27246 & 142.41 & 29720 \\
\hline 420 & 119.99 & 29613 & 148.19 & 32626 \\
\hline 440 & 123.11 & 32045 & 153.85 & 35647 \\
\hline 460 & 126.05 & 34537 & 159.38 & 38780 \\
\hline 480 & 128.81 & 37087 & 164.81 & 42022 \\
\hline 497 & 131.03 & 39262 & 169.33 & 44895 \\
\hline
\end{tabular}


films, as shown in Fig. 11. As based on DSC measurements of heats of fusion, the quenched films may originally contain a crystallinity of about 0.25 , before additional crystallization starts at temperatures just above the glass transition temperature.

The liquidus heat capacity, from $335 \mathrm{~K}$ in the supercooled liquid state to $620 \mathrm{~K}$ in the melt, was obtained by combining the adiabatic measurement of the quenched film from 335 to $370 \mathrm{~K}$ adjusted for the $\Delta C_{p}$ due to a residual crystalline content in the original film and the DSC measurements of molten PCTFE above $495 \mathrm{~K}$ using the short interval scan as mentioned earlier. The trend of the liquidus $C_{p}$ behavior of a low molecular weight wax as observed with DSC was used as a guide to the functionality between the two measurements. The assigned liquidus heat capacity is also shown in Fig. 11.

3.3.2 Thermodynamic Functions and Residual Entropy The thermodynamic functions for the crystalline PCTFE are listed in Table 4. The functions are calculated based on the estimated crystalline heat capacity as mentioned above, with the assumption that there is no residual entropy at $0 \mathrm{~K}$ for the crystalline phase. If there is any residual entropy for the crystal, the free energy term should be corrected by an amount equal to $T S_{0}$.

The thermodynamic functions of the amorphous phase and the liquid are listed in Table 5. These functions are calculated based on the estimated amorphous and liquid heat capacity as mentioned above. The integration for the enthalpy and entropy are based on the zero-point enthalpy and entropy of the glass rather than the crystal. As the amorphous phase is expected to have a residual entropy at $0 \mathrm{~K}$, the free energy is not calculated.

The heat of fusion of the crystalline state is considered to be about $40 \mathrm{~J} \mathrm{~g}^{-1}$ or $4.7 \mathrm{~kJ} \mathrm{~mol}^{-1}$ at 497 $\mathrm{K}$. The corresponding entropy of fusion is $9.3 \mathrm{~J} \mathrm{~K}^{-1}$ $\mathrm{mol}^{-1}$. These values will yield $\left(H_{\mathrm{liq}}-H_{\mathrm{x}}\right)_{497}$ of 44.0 $\mathrm{kJ} \mathrm{mol}{ }^{-1}$ and $S_{497, \text { iq }}$ of $179.5 \mathrm{~J} \mathrm{~K}^{-1} \mathrm{~mol}^{-1}$. Then $\left(H_{\mathrm{gl}}-H_{\mathrm{x}}\right)_{0}$ will be about $1.6 \mathrm{~kJ} \mathrm{~mol}^{-1}$ and the residual entropy for the amorphous PCTFE at $0 \mathrm{~K}, S_{0, \mathrm{~g}}$, will be about $2.0 \mathrm{~J} \mathrm{~K}^{-1} \mathrm{~mol}^{-1}$. This $S_{0, \mathrm{gl}}$ value, although reasonable, is smaller than the usual observation mentioned for a collection of reliable residual entropies [26,32] of around $(R \ln 2) / 2$ or 3 $J K^{-1}$ per each chain atom or bead. The smaller residual entropy may be the result of the disorder existing in the crystalline PCTFE or a low estimation of the heat of fusion of the PCTFE crystal.
Table 5. Thermodynamic functions of amorphous and liquidus PCTFE (Units in J, K, and mol. $1 \mathrm{~mol}=116.47 \mathrm{~g}$ )

\begin{tabular}{|c|c|c|c|}
\hline $\begin{array}{l}T \\
\mathrm{~K}\end{array}$ & $\begin{array}{c}C_{p} \\
\mathrm{~J} \mathrm{~K}^{-1} \mathrm{~mol}^{-1}\end{array}$ & $\begin{array}{c}H-H_{0, \mathrm{gl}} \\
\mathrm{J} \mathrm{mol}^{-1}\end{array}$ & $\begin{array}{c}S-S_{0, \mathrm{gl}} \\
\mathrm{J} \mathrm{K}^{-1} \mathrm{~mol}^{-1}\end{array}$ \\
\hline 5 & 0.69 & 0.95 & 0.26 \\
\hline 10 & 2.74 & 9.20 & 1.32 \\
\hline 15 & 5.16 & 28.90 & 2.88 \\
\hline 20 & 7.59 & 60.82 & 4.70 \\
\hline 30 & 12.09 & 159.65 & 8.65 \\
\hline 40 & 16.24 & 301.47 & 12.70 \\
\hline 50 & 20.30 & 484.16 & 16.60 \\
\hline 60 & 24.39 & 707.57 & 20.82 \\
\hline 70 & 28.54 & 972.18 & 24.89 \\
\hline 80 & 32.71 & 1278.4 & 28.98 \\
\hline 90 & 36.83 & 1626.2 & 33.07 \\
\hline 100 & 40.84 & 2014.7 & 37.16 \\
\hline 120 & 48.39 & 2908.0 & 45.28 \\
\hline 140 & 55.04 & 3943.5 & 53.25 \\
\hline 160 & 61.34 & 5107.8 & 61.01 \\
\hline 180 & 67.37 & 6395.3 & 68.59 \\
\hline 200 & 73.14 & 7800.9 & 75.99 \\
\hline 220 & 78.65 & 9319.2 & 83.22 \\
\hline 240 & 83.90 & 10945 & 90.29 \\
\hline 260 & 88.89 & 12673 & 97.20 \\
\hline 273.15 & 93.00 & 13869 & 101.68 \\
\hline 280 & 95.59 & 14514 & 104.02 \\
\hline 298.15 & 103.93 & 16323 & 110.27 \\
\hline 300 & 104.90 & 16515 & 110.92 \\
\hline 320 & 116.81 & 18729 & 118.06 \\
\hline 335 & 127.43 & 20559 & 123.65 \\
\hline 340 & 127.97 & 21198 & 125.54 \\
\hline 360 & 130.04 & 23778 & 132.91 \\
\hline 380 & 131.98 & 26399 & 139.99 \\
\hline 400 & 133.79 & 29056 & 146.81 \\
\hline 420 & 135.46 & 31749 & 153.38 \\
\hline 440 & 137.01 & 34474 & 159.72 \\
\hline 460 & 138.42 & 37229 & 165.84 \\
\hline 480 & 139.70 & 40010 & 171.76 \\
\hline 497 & 140.68 & 42393 & 176.64 \\
\hline 500 & 140.84 & 42816 & 177.48 \\
\hline 520 & 141.86 & 45643 & 183.03 \\
\hline 540 & 142.74 & 48489 & 188.40 \\
\hline 560 & 143.49 & 51352 & 193.60 \\
\hline 580 & 144.10 & 54228 & 198.65 \\
\hline 600 & 144.59 & 57115 & 203.54 \\
\hline 620 & 144.94 & 60010 & 208.29 \\
\hline
\end{tabular}

\section{Conclusion}

One advantage of adiabatic calorimetry over DSC is the ability to detect the direction and to separate the effects of rela tively long term spontaneous thermal events originating in the sample. The DSC measurement provides only a single overall heatflow thermogram as a function of time during a scan. The adiabatic calorimetry allows the extraction of short term equilibrium heat capacities such 
as contributions from lattice vibrations from long term spontaneous thermal events such as relaxation and crystallization, by observing the adiabatic temperature drifts as a function of time. Magnitudes, directions, and locations of the spontaneous event may also be measured by adiabatic calorimetry. Differential scanning calorimetry is however more advantageous if large numbers of small-size samples need to be tested at a lower precision.

The lattice heat capacities of PCTFE samples of different crystallinities clearly show a glass transition in the region from 320 to $350 \mathrm{~K}$, heretofore undetected by other thermal measurements. The spontaneous drifts observed in adiabatic calorimetry have also confirmed the glass-like relaxation phenomenon, which give rise to a positive temperature drift for quenched glasses and negative drift for annealed glasses.

The glass transition temperature is, however, lowered for samples of higher crystallinity. This may be caused by the rejection of the uncrystallizable portion of a polymer chain, which itself may have a lower glass transition temperature than the overall glass transition temperature as contributed by all different structures.

Although a few approximations were applied to derive the thermodynamic functions for PCTFE crystal, glass, and liquid, the small residual entropy of the glass may be the result of a disorder in the crystalline structure. Heat capacities of the highly crystalline sample behaved almost identically to that of a low crystallinity sample in the cryogenic temperature region down to $2.5 \mathrm{~K}$, where a region for the Debye $T^{3}$ law was not yet reached.

The melting range of PCTFE is very wide, with the majority of the melting occurring in a range of $60 \mathrm{~K}$. For ordinary samples with a smooth cooling history and having a crystallinity around 0.5 , annealing at any temperature above $240 \mathrm{~K}$ may cause a reorganization into a non-smooth distribution of crystallite sizes. By successively annealing at lower temperatures, a sample with more than five melting peaks was prepared.

\section{References}

[1] J. D. Hoffman, J. Am. Chem. Soc. 74, 1696 (1952).

[2] W. Reese and J. E. Tucker, J. Chem. Phys. 43, 105 (1965).

[3] W. K. Lee, P. C. Lau, and C. L. Choy, Polymer 15, 487 (1974).

[4] B. Terziiska, H. Madge, and V. Lovtchinov, J. Thermal Anal. 20, 33 (1981).

[5] L. Mandelkern, G. M. Martin, and F. A. Quinn, J. Res. Natl. Bur. Stand. (U.S.) 58, 137 (1957).
[6] J. D. Hoffman and J. J. Weeks, J. Res. Natl. Bur. Stand. (U.S.) 60, 465 (1958).

[7] J. D. Hoffman, G. Williams, and E. Passaglia, J. Polym. Sci. C14, 173 (1966).

[8] E. Sacher, J. Polym. Sci., Polym. Lett. Ed. 18, 333 (1980).

[9] J. M. Crissman and E. Passaglia, J. Polym. Sci. C14, 237 (1966).

[10] S. S. Chang, Proc. 7th Symp. Thermophys. Prop., ASME, 75 (1977).

[11] S. S. Chang, J. Polym. Sci. C43, 43 (1973).

[12] H. S. Kaufman, J. Am. Chem. Soc. 75, 1477 (1953).

[13] L. G. Roldan and H. S. Kaufman, Norelco Reporter 10, No. 1, 11 (1963).

[14] Z. Mencik, J. Polym. Sci., Polym. Phys. Ed. 11, 1589 (1973).

[15] K. F. Sterrett, D. H. Blackburn, A. B. Bestul, S. S. Chang, and J. Horman, J. Res. Natl. Bur. Stand. (U.S.) 69C, 19 (1965).

[16] S. S. Chang, J. Res. Natl. Bur. Stand. (U.S.) 80A, 669 (1976).

[17] H. Diesselhorst, Z. Instrumentkunde 26, 297 (1906).

[18] S. S. Chang, Rev. Sci. Instrum. 40, 822 (1966).

[19] R. E. Bedford, M. Durieux, R. Muijlwijk, and C. R. Barber, Metrologia 5, 47 (1969).

[20] H. Plumb and G. Cataland, Metrologia 2, 127 (1966).

[21] F. L. McCrackin and S. S. Chang, Rev. Sci. Instrum. 46, 550 (1975).

[22] H. Preston-Thomas, Metrologia 27, 3 (1990).

[23] S. S. Chang, Proc. 7th Intl. Symp. Thermophys. Prop., ASME, 83 (1977).

[24] D. A. Ditmars, S. Ishihara, S. S. Chang, G. Bernstein, and E. D. West, J. Res. Natl. Bur. Stand. (U.S.) 87, 159 (1982).

[25] S. S. Chang and A. B. Bestul, J. Res. Natl. Bur. Stand. (U.S.) 75A, 113 (1971).

[26] S. S. Chang and A. B. Bestul, J. Chem. Thermodyn. 6, 325 (1974).

[27] J. C. Lasjaunias, C. R. Acad. Sci. Ser. B 269, 763 (1969).

[28] S. S. Chang, Thermal Anal. Polym. Characterization, E. A. Turi, ed., Heyden $\&$ Sons (1981) p. 98.

[29] S. S. Chang, J. Polym. Sci., Polym. Symp. 71, 59 (1984).

[30] C. L. Choy, R. G. Hunt, and G. L. Salinger, J. Chem. Phys. 52, 3629 (1972).

[31] P. Zoller, D. L. Fehl, and J. R. Dillinger, J. Polym. Sci., Polym. Phys. Ed. 11, 1441 (1973).

[32] S. S. Chang and A. B. Bestul, J. Chem. Phys. 56, 503 (1972).

[33] S. S. Chang, J. Res. Natl. Bur. Stand. (U.S.) 82, 9 (1977).

[34] S. S. Chang, J. Res. Natl. Bur. Stand. (U.S.) 78a, 387 (1974).

[35] S. S. Chang, J. Chem. Phys. 79, 6229 (1983).

[36] R. J. Graves and H. W. White, Cryogenics 20, 525 (1980).

[37] C. M. Guttman, to be published.

[38] S. S. Chang, J. A. Horman, and A. B. Bestul, J. Res. Natl. Bur. Stand. (U.S.) 71A, 293 (1967).

[39] A. H. Scott, D. J. Scheiber, A. J. Curtis, J. I. Lauritzen, Jr., and J. D. Hoffman, J. Res. Natl. Bur. Stand. (U.S.) 66a, 269 (1962).

[40] A. P. Gray and K. Casey, J. Polym. Sci., Polym. Lett. Ed. 2, 381 (1964).

[41] S. S. Chang, Polym. Comm. 29, 138 (1988).

[42] A. M. Bueche, J. Am. Chem. Soc. 74, 65 (1952).

[43] F. P. Price, J. Chem. Phys. 19, 973 (1951).

[44] J. D. Hoffman and J. J. Weeks, J. Res. Natl. Bur. Stand. (U.S.) 66A, 13 (1962).

[45] H. T. Hall, J. Am. Chem. Soc. 74, 68 (1952). 
Journal of Research of the National Institute of Standards and Technology

About the authors: Shu-Sing Chang is a Research Chemist in the Polymers Division at NIST. James J. Weeks is a Research Chemist who retired from the National Bureau of Standards (now NIST). The National Institute of Standards and Technology is an agency of the Technology Administration, U.S. Department of Commerce. 